\title{
The Division Problem with Voluntary Participation*
}

\author{
Gustavo Bergantiños ${ }^{\dagger}$, Jordi Massó ${ }^{\ddagger}$, and Alejandro Neme ${ }^{\S}$
}

April 2009

\begin{abstract}
The division problem consists of allocating a given amount of an homogeneous and perfectly divisible good among a group of agents with single-peaked preferences on the set of their potential shares. A rule proposes a vector of shares for each division problem. The literature has implicitly assumed that agents will find acceptable any share they are assigned to. In this paper we consider the division problem when agents' participation is voluntary. Each agent has an acceptable interval of shares where his preferences are single-peaked. A rule has to propose to each agent either an acceptable share or the zero share because otherwise, he would prefer to exclude himself and leave with the zero share; this would require to reassign the remaining agents' shares. We study a subclass of efficient and consistent rules and characterize extensions of the uniform rule that deal explicitly with agents' voluntary participation.
\end{abstract}

${ }^{*}$ We thank Bettina Klaus for helpful comments. The work of G. Bergantiños is partially supported by research grant ECO2008-03484-C02-01 from the Spanish Ministry of Science and Innovation and FEDER. Support for the research of J. Massó was received through the prize "ICREA Acadèmia" for excellence in research, funded by the Generalitat de Catalunya. He also acknowledges the support of the Barcelona Graduate School of Economics, where he is an Affiliated Professor, through its Research Recognition Programme. His work is also supported by the Spanish Ministry of Science and Innovation through grants ECO2008-04756 (Grupo Consolidado-C) and CONSOLIDER-INGENIO 2010 (CDS2006-00016), and by the Generalitat de Catalunya through grant SGR2005-00454. The work of A. Neme is partially supported by the Universidad Nacional de San Luis through grant 319502, by the Consejo Nacional de Investigaciones Científicas y Técnicas CONICET, through grant PICT-02114, and by the Agencia Nacional de Promoción Científica y Técnica, through grant 03-10814.

${ }^{\dagger}$ Research Group in Economic Analysis. Facultade de Económicas, Universidade de Vigo. 36310, Vigo (Pontevedra), Spain. E-mail: gbergant@uvigo.es

‡Departament d’Economia i d'Història Econòmica and CODE. Universitat Autònoma de Barcelona. 08193, Bellaterra (Barcelona), Spain. E-mail: jordi.masso@uab.es

§Instituto de Matemática Aplicada de San Luis. Universidad Nacional de San Luis and CONICET. Ejército de los Andes 950. 5700, San Luis, Argentina. E-mail: aneme@unsl.edu.ar 
Journal of Economic Literature Classification Number: D71.

Keywords: Division Problem, Single-peaked Preferences, Uniform Rule, Voluntary Participation.

\section{Introduction}

The division problem consists of a set of agents that have to share an amount of an homogeneous and perfectly divisible good. Each agent has single-peaked preferences on the set of his potential shares; namely, there is an amount of the good (the peak of the agent) that is his most-preferred share and in both sides of the peak the preference is monotonic, decreasing at its right and increasing at its left. Since preferences reflect idiosyncratic characteristics of the agents, they have to be elicited by a rule that maps each division problem (a set of agents, a preference profile of declared list of single-peaked preferences, one for each agent, and the amount of the good to be allocated) into a vector of shares. But in general, the sum of the peaks will be either larger or smaller than the total amount to be allocated. A positive or negative rationing problem emerges depending on whether the sum of the peaks exceeds or fails short the fixed amount. Rules differ from each other in how this rationing problem is resolved in terms of incentives, efficiency, fairness, monotonicity, consistency, etc.

There are many examples of allocation problems that fit with this general description. For instance, a group of agents participate in an activity that requires a fixed amount of labor (measured in units of time). Agents have a maximal number of units of time to contribute and consider working as being undesirable. Suppose that labor is homogeneous and the wage is fixed. Then, strictly monotonic and quasi-concave preferences on the set of bundles of money and leisure generate single-peaked preferences on the set of potential shares where the peak is the amount of working time associated to the optimal bundle. Similarly, a group of agents join a partnership to invest in a project (an indivisible bond with a face value, for example) that requires a fixed amount of money (neither more nor less). Their risk attitudes and wealth induce single-peaked preferences on the amount to be invested. In both cases, a rule is required to solve the rationing problem arising from a vector of peaks that do not add up the needed amount.

However, in many applications (like those described above), agents' participation can not be compulsory. Hence, the rule can not propose to an agent an unacceptable share; otherwise, the agent would prefer to not participate at all and leave with the zero share. In this paper we study rules that solve the rationing problem when agents' participation is voluntary. We call an allocation problem of this type, a division problem with voluntary 
participation (a problem, for short). Now, in a problem each agent's preferences are characterized by an interval of acceptable shares where preferences are single-peaked. Only shares inside this interval are considered to be acceptable. A rule will have to propose, for each problem, a vector where each agent receives either an acceptable share or zero. Now the vector of zero shares to everybody is a feasible allocation.

We are interested in rules that satisfy a set of desirable properties. First, efficiency. A rule is efficient if it always selects Pareto optimal allocations. Efficiency guarantees that in solving the rationing problem (either positive or negative) no amount of the good is wasted. Second, consistency. A rule is consistent if the proposed shares at a given problem coincide with the shares that the rule would propose at any smaller problem obtained after that a subset of agents, agreeing with the amounts the rule has assigned to them, leave the society taking with them their already assigned shares. Consistency guarantees that, in order to follow the rule's prescription at the reduced problem, the remaining agents do not have to reallocate their shares. Third, individual rationality from equal division. Suppose that we assign to each agent his smallest acceptable share. The rest is divided as equally as possible under the condition that no agent receives more than his largest acceptable share. A rule satisfies this property by choosing a Pareto improvement from the previous allocation. ${ }^{1}$ Individual rationality from equal division embeds to the rule a minimal egalitarian principal only broken for two reasons. First, to keep biding the restrictions derived from the requirement that agents have to receive acceptable shares and second, to admit Pareto improvements from this egalitarian allocation. In contrast with the division problem when all shares are acceptable, ${ }^{2}$ we show that when agents' participation is voluntary the fundamental properties of efficiency, anonymity and strategy-proofness are incompatible. ${ }^{3}$ In particular, strategy-proofness and efficiency together makes the rule to be too stringent since efficiency requires it to be sensitive to agents' participation intervals

\footnotetext{
${ }^{1}$ See Sönmez (1994) for an analysis of rules satisfying this property in the context of division problems with compulsory participation.

${ }^{2}$ In this setting Sprumont (1991) characterizes the uniform rule as the unique rule satisfying efficiency, anonymity (the names of the agents do not play any role), and strategy-proofness (truth-telling is a dominant strategy in the direct revelation game induced by the rule).

${ }^{3}$ Specifically, strategy-proofness is incompatible with a much weaker requirement of efficiency (see Subsection 3.1 for its formal definition). Moreover, strategy-proofness is incompatible with anonymity in the subclass of reasonable rules (those that satisfy the minimal requirement that if the sum of all ideal shares is equal to the amount of the good to be allocated, the proposed shares coincide with the ideal shares). Finally, efficiency is also incompatible with anonymity. In Subsection 3.2 we give formal proofs of these incompatibilities.
} 
but then it would respond too much to agents' preferences, becoming easily manipulable. ${ }^{4}$ We proceed by leaving aside incentive issues and by focusing on the class of efficient and consistent rules that are individually rational from equal division.

Before moving to the general description of our results we want to stress a fundamental attribute of rules when applied to division problems with voluntary participation. Fix a problem (a set of agents, their preferences, and the amount of the good to be allocated). A rule has to make two choices. First, it has to select a subset of agents (a coalition) among whom the good will be allocated. This coalition has to be admissible for the problem: it should be possible to allocate the total amount of the good among its members without harming their participation constraints. Second, and given this chosen coalition (if nonempty), the rule has to select (among potentially many) a particular share allotted to each of its members. When participation is compulsory rules disregard the first issue and select always the grand coalition. In this setting the uniform rule has emerged as the most appealing one. ${ }^{5}$ At each division problem with compulsory participation the uniform rule tries to allocate the amount of the good among all agents as equally as possible, keeping the efficiency constraints binding. Hence, all agents are constrained in the same way; i.e., either each receives a share below his peak (when the sum of all their ideals is larger than the total amount) or each agent receives a share above his peak (when the sum of all their ideals is smaller than the total amount).

Our results identify axiomatically three nested classes of rules. In all cases the set of axioms will single out a unique way of allocating the amount of the good among the members of an admissible chosen coalition. The classes will differ precisely on how their elements choose the admissible coalition. This unique allotting way consists of the following natural extension of the uniform rule. Fix a problem. If no coalition is admissible, assign the zero share to all agents. Otherwise, take the chosen non-empty admissible coalition. Then the allocation of the good among its members can be described as a two steps procedure. First, assign to each agent in the coalition his smallest acceptable share. The remainder is assigned by adding uniformly the same amount to every agent in the coalition. If the sum of the peaks exceeds the amount to be allocated then the rule stops adding to those agents whose peak is reached, and keeps adding uniformly to the rest. Observe that

\footnotetext{
${ }^{4}$ In contrast again, Barberà, Jackson and Neme (1997) shows that when agents' participation is compulsory the class of strategy-proof and efficient rules is extremely large.

${ }^{5}$ See Ching (1992, 1994), Schummer and Thomson (1997), Sönmez (1994), Sprumont (1991), Thomson $(1994,1995,1997)$, and Weymark (1999) for alternative characterizations of the uniform rule in the division problem. In the surveys on strategy-proofness of Barberà (1996, 2001), Jackson (2001) and Sprumont (1995) the division problem and the uniform rule plays a prominent role.
} 
in this case the remainder will eventually be exhausted before all peaks are reached. If the sum of the peaks is smaller than the amount to be allocated then the rule also keeps adding uniformly to all agents, and stops adding only to those agents whose largest acceptable share is reached, and keeps adding uniformly to the rest. Observe now that since the coalition was admissible the remainder will eventually be exhausted before reaching all largest acceptable shares. We call any rule satisfying this allotment procedure an extended uniform rule. There are many because at many problems there are many admissible coalitions. Hence, extended uniform rules differ only on the choice of the subset of agents among whom the amount of the good is allocated.

Theorem 1 characterizes the class of efficient, consistent and individually rational from equal division rules as the subset of extended uniform rules that select the admissible coalition by choosing coherently the full set of agents whenever it is possible. Theorem 2 characterizes the subclass of rules that, in addition to the previous properties, satisfy an independence of irrelevant alternatives like property (that we call admissible contraction). This class consists of the subset of extended uniform rules that at each problem choose the admissible coalition by maximizing a given monotonic order on the set of all finite coalitions. Theorem 3 characterizes the smaller subclass of rules that in addition to efficiency, consistency, and individually rationality form equal division also satisfy order preservation with respect to a given order of priority among individual agents. This class consists of the subset of extended uniform rules that at each problem choose the admissible coalition by selecting lexicographically according to the given order. We also show that in all three characterizations the axioms are independent.

The paper is organized as follows. In Section 2 we describe the model. In Section 3 we define several properties that a rule may satisfy and show some basic incompatibilities among them. In Section 4 we define extended uniform rules. In Section 5 we present the main results of the paper. In Section 6 we conclude with some final remarks. Three appendices at the end of the paper collect the proofs of the three theorems.

\section{The model}

Let $t>0$ be a fixed amount of an homogeneous and perfectly divisible good. A finite set of agents is considering the possibility of dividing $t$ among a subset of them, to be determined according to their preferences. Since we will be considering situations where the amount of the good $t$ and the finite set of agents may vary, let $\mathbb{N}$ be the set of positive integers and let $\mathcal{N}$ be the family of all non-empty and finite subsets of $\mathbb{N}$. The set of agents is then $N \in \mathcal{N}$ with cardinality $n$. In contrast with Sprumont (1991), we consider situations 
where each agent has the right to exclude himself from the division problem (a feasible allocation is that the good is not divided at all) and obtain a share equal to zero. Thus, and since each agent $i$ can not be forced to receive an unacceptable share of the good, his preferences $\succeq_{i}$ are defined on the set $\{0\} \cup\left[l_{i}, u_{i}\right]$, where $\left[l_{i}, u_{i}\right] \subseteq[0,+\infty]$ is agent $i$ 's interval of acceptable shares. We assume that $\succeq_{i}$ is a complete, reflexive, and transitive binary relation on $\{0\} \cup\left[l_{i}, u_{i}\right]$. Given $\succeq_{i}$ let $\succ_{i}$ be the antisymmetric binary relation induced by $\succeq_{i}$ (i.e., for all $x_{i}, y_{i} \in\{0\} \cup\left[l_{i}, u_{i}\right], x_{i} \succ_{i} y_{i}$ if and only if $y_{i} \succeq x_{i}$ does not hold) and let $\sim_{i}$ be the indifference relation induced by $\succeq_{i}$ (i.e., for all $x_{i}, y_{i} \in\{0\} \cup\left[l_{i}, u_{i}\right]$, $x_{i} \sim_{i} y_{i}$ if and only if $x_{i} \succeq_{i} y_{i}$ and $\left.y_{i} \succeq x_{i}\right)$. We will also assume that $\succeq_{i}$ is single-peaked on $\left[l_{i}, u_{i}\right]$ and we will denote by $p_{i} \in\left[l_{i}, u_{i}\right]$ agent $i$ 's peak. Formally, agent $i$ 's preferences $\succeq_{i}$ is a complete preorder on the set $\{0\} \cup\left[l_{i}, u_{i}\right]$ that satisfies the following additional properties:

(P.1) there exists $p_{i} \in\left[l_{i}, u_{i}\right]$ such that $p_{i} \succ_{i} x_{i}$ for all $x_{i} \in\left[l_{i}, u_{i}\right] \backslash\left\{p_{i}\right\}$;

(P.2) $x_{i} \succ_{i} y_{i}$ for any pair of shares $x_{i}, y_{i} \in\left[l_{i}, u_{i}\right]$ such that either $y_{i}<x_{i} \leq p_{i}$ or $p_{i} \leq x_{i}<y_{i}$; and

(P.3) $x_{i} \succ_{i} 0$ for all $x_{i} \in\left[l_{i}, u_{i}\right] \backslash\{0\}$.

Observe that agent $i$ 's preferences are defined on $\{0\} \cup\left[l_{i}, u_{i}\right]$ and are independent of $t$. Moreover, we are admitting the possibilities that $l_{i}=0$ and $l_{i}=p_{i}=u_{i}$. A preference $\succeq_{i}$ of agent $i$ is (partly) characterized by the triple $\left(l_{i}, p_{i}, u_{i}\right)$. There are many preferences of agent $i$ with the same $\left(l_{i}, p_{i}, u_{i}\right)$; however, they differ only on how two shares on different sides of $p_{i}$ are ordered while all of them coincide on the ordering on the shares on each of the sides of $p_{i}$. This multiplicity will often be irrelevant. A profile $\succeq_{N}=\left(\succeq_{i}\right)_{i \in N}$ is an $n$-tuple of preferences satisfying properties (P.1), (P.2) and (P.3) above. Given a profile $\succeq_{N}$ and agent $i$ 's preferences $\succeq_{i}^{\prime}$ we denote by $\left(\succeq_{i}^{\prime}, \succeq_{N \backslash\{i\}}\right)$ the profile where $\succeq_{i}$ has been replaced by $\succeq_{i}^{\prime}$ and all other agents have the same preferences. When no confusion arises we denote the profile $\succeq_{N}$ by $\succeq$.

A division problem with voluntary participation (a problem for short) is a triple $(N, \succeq, t)$ where $N$ is the set of agents, $\succeq$ is a profile and $t$ is the amount of the good to be divided. Let $\mathcal{P}$ be the set of all problems. A problem where for all agents their participation is compulsory and preferences are single-peaked on $[0,+\infty)$ is known as the division problem (see Ching and Serizawa (1994)); i.e., for all $i \in N, l_{i}=0, u_{i}=+\infty$ and (P.1) and (P.2) hold. Observe that in a division problem agent $i$ may strictly prefer the 0 share to some strictly positive share. Thus, if participation is voluntary and $l_{i}=0$ then, either $u_{i}=0$ or else $x_{i} \succ_{i} 0$ for all $x_{i} \in\left(0, u_{i}\right]$ (that is, (P.3) holds). 
The set of feasible allocations of problem $(N, \succeq, t)$ is

$F A(N, \succeq, t)=\left\{\left(x_{1}, \ldots, x_{n}\right) \in \mathbb{R}_{+}^{N} \mid \sum_{j \in N} x_{j} \in\{0, t\}\right.$ and for each $\left.i \in N, x_{i} \in\{0\} \cup\left[l_{i}, u_{i}\right]\right\}$

Note that this set is never empty since the allocation $(0, \ldots, 0) \in \mathbb{R}_{+}^{N}$ is always feasible. Moreover, there are problems for which $(0, \ldots, 0)$ is the unique feasible allocation; for instance the problem $(N, \succeq, t)$ where $N=\{1,2\}, t=10$, and $\succeq_{1}$ and $\succeq_{2}$ are characterized by $\left(l_{1}, p_{1}, u_{1}\right)=\left(l_{2}, p_{2}, u_{2}\right)=(1,2,3)$.

A coalition $S \subseteq N$ is admissible (at profile $\succeq$ and amount $t$ ) if it is either empty or it is possible to divide $t$ among the agents in $S$ according to their preferences; namely, coalition $S \neq \varnothing$ is admissible at $(N, \succeq, t)$ if there exists $x=\left(x_{j}\right)_{j \in S} \in \mathbb{R}_{+}^{S}$ such that $\sum_{j \in S} x_{j}=t$ and $l_{i} \leq x_{i} \leq u_{i}$ for all $i \in S$. It is obvious that $S \neq \varnothing$ is admissible if and only if $\sum_{j \in S} l_{j} \leq t \leq \sum_{j \in S} u_{j}$. We denote by $A C(N, \succeq, t)$ the set of all admissible coalitions at $(N, \succeq, t)$. Namely,

$$
A C(N, \succeq, t)=\{S \subseteq N \mid S \text { is admissible at }(N, \succeq, t)\} .
$$

Observe that $A C(N, \succeq, t)$ is never empty because it always contains the empty coalition.

A rule $f$ assigns to each problem in $\mathcal{P}$ a feasible allocation; that is, $f(N, \succeq, t) \in$ $F A(N, \succeq, t)$ for all $(N, \succeq, t) \in \mathcal{P}$. Hence, a rule $f$ can be seen as a systematic way of assigning to each $(N, \succeq, t) \in \mathcal{P}$ the two different aspects of the solution of the problem. First, the admissible coalition $S \in A C(N, \succeq, t)$. If $S \neq \varnothing$ we denote it by

$$
c^{f}(N, \succeq, t)=\left\{i \in N \mid f_{i}(N, \succeq, t) \in\left[l_{i}, u_{i}\right]\right\}
$$

Obviously, if $i \notin c^{f}(N, \succeq, t)$ then $f_{i}(N, \succeq, t)=0$. Second, how the amount $t$ is divided among the members of $c^{f}(N, \succeq, t)$; i.e.,

$$
t=\sum_{j \in c^{f}(N, \succeq, t)} f_{j}(N, \succeq, t) .
$$

We will later see that to identify rules satisfying appealing properties we may have some freedom when choosing one among the set of admissible coalitions while the properties will determine a unique way of dividing the amount of the good.

\section{Properties of rules}

\subsection{Definitions}

In this subsection we define several properties that a rule may satisfy. 
Rules require each agent to report a preference. A rule is strategy-proof if it is always in the best interest of agents to reveal their preferences truthfully; namely, it induces truth-telling as a dominant strategy in the direct revelation game generated by the rule.

(Strategy-Proofness) A rule $f$ is strategy-proof if for each $\left(N, \succeq_{N}, t\right), i \in N$, and $\succeq_{i}^{\prime}$,

$$
f_{i}\left(N, \succeq_{N}, t\right) \succeq_{i} f_{i}\left(N,\left(\succeq_{i}^{\prime}, \succeq_{N \backslash\{i\}}\right), t\right)
$$

Given a problem $\left(N, \succeq_{N}, t\right)$ we say that agent $i \in N$ manipulates $f$ at profile $\succeq_{N}$ via $\succeq_{i}^{\prime}$ if $f_{i}\left(N,\left(\succeq_{i}^{\prime}, \succeq_{N \backslash\{i\}}\right), t\right) \succ_{i} f_{i}\left(N, \succeq_{N}, t\right)$. Thus, a rule $f$ is strategy-proof if no agent can manipulate it at any profile.

A rule is anonymous if it only depends on the characteristics of the profile and not on the name of the agents having the corresponding preference; that is, it is invariant with respect to the index given to the agents. Let $\sigma: \mathbb{N} \rightarrow \mathbb{N}$ be a one-to-one mapping, $N$ a set of agents and $\succeq_{N}$ a profile. Define the set of agents $\sigma(N)=\{j \in \mathbb{N} \mid$ there exists $i \in N$ such that $\sigma(i)=j\}$ and the profile $\sigma\left(\succeq_{N}\right)=\left(\sigma\left(\succeq_{N}\right)_{j}\right)_{j \in \sigma(N)}$ by letting $\sigma\left(\succeq_{N}\right)_{\sigma(i)}=\succeq_{i}$ for each $i \in N$.

(Anonymity) A rule $f$ is anonymous if for any one-to-one mapping $\sigma: \mathbb{N} \rightarrow \mathbb{N}$ and any problem $\left(N, \succeq_{N}, t\right), f_{i}\left(N, \succeq_{N}, t\right)=f_{\sigma(i)}\left(\sigma(N), \sigma\left(\succeq_{N}\right), t\right)$ for all $i \in N$.

A rule is efficient if it always selects a Pareto optimal allocation.

(EFFICIENCY) A rule $f$ is efficient if for each problem $(N, \succeq, t)$ there is no feasible allocation $\left(y_{j}\right)_{j \in N} \in F A(N, \succeq, t)$ with the property that $y_{i} \succeq_{i} f_{i}(N, \succeq, t)$ for all $i \in N$ and $y_{j} \succ_{j} f_{j}(N, \succeq, t)$ for some $j \in N$.

A rule is weak efficient if it distributes the amount $t$ whenever there exists a feasible allocation that does it.

(Weak Efficiency) A rule $f$ is weak efficient if for each problem $(N, \succeq, t)$ such that there exists a feasible allocation $\left(y_{j}\right)_{j \in N} \in F A(N, \succeq, t)$ with the property that $\sum_{j \in N} y_{j}=t$, then $\sum_{j \in N} f_{j}(N, \succeq, t)=t$.

A rule is reasonable if it satisfies the minimum requirement of efficiency saying that if the sum of the peaks of the agents is equal to the amount $t$ then, the share of each agent has to be equal to his peak.

(REasonability) A rule $f$ is reasonable if for each problem $(N, \succeq, t)$ such that $\sum_{j \in N} p_{j}=$ $t$ then, $f_{i}(N, \succeq, t)=p_{i}$ for all $i \in N{ }^{6}$

\footnotetext{
${ }^{6}$ Notice that if $f$ is efficient then, $f$ is weakly efficient and reasonable.
} 
A rule is consistent if the following requirement holds. Apply the rule to a given problem and assume that a subset of agents leave with their corresponding shares. Consider the new problem formed by the set of agents that remain with the same preferences that they had in the original problem and the total amount of the good minus the sum of the shares received by the subset of agents that already left. Then, the rule does not require to reallocate the shares of the remaining agents.

(Consistency) A rule $f$ is consistent if for each problem $\left(N, \succeq_{N}, t\right)$, each non-empty subset of agents $S \subset N$, and each $i \in S$,

$$
f_{i}\left(N, \succeq_{N}, t\right)=f_{i}\left(S, \succeq_{S}, t-\sum_{j \in N \backslash S} f_{j}\left(N, \succeq_{N}, t\right)\right)
$$

For the division problem with compulsory participation Sönmez (1994) proposed the principle of individual rationality from equal division. A rule $f$ is individually rational from equal division if all agents receive a share that is at least as good as the equal division share; namely, for each division problem $(N, \succeq, t)$,

$$
f_{i}(N, \succeq, t) \succeq_{i} \frac{t}{n}
$$

for all $i \in N$. In a division problem equal division is always feasible but often is not efficient. Precisely, this principle tries to make compatible equal division with efficiency by allowing for Pareto improvements from the equal division share. Observe that in our setting the allocation $\left(\frac{t}{n}, \ldots, \frac{t}{n}\right)$ may not be feasible and/or there may not even exist a vector of shares at which all agents are better off than at equal division. Thus, when agents' participation is voluntary, this property is too strong (no rule satisfies it) and it can not be applied directly. However, and since we think that its content is appealing we suggest to use the same principle as follows. Assume that in the problem $(N, \succeq, t)$ the coalition $N$ is admissible. Preliminarily assign to each agent $i$ the amount $l_{i}$ (which is possible since $N$ is admissible). The remaining amount $t-\sum_{j \in N} l_{j}$ has still to be allocated, but again, by feasibility, each agent $i$ must receive overall at most $u_{i}$. Then, allocate the remaining amount $t-\sum_{j \in N} l_{j}$ as equally as possible, but making sure that no agent $i$ receives additionally more than $u_{i}-l_{i}$. Each agent must receive a share at least as good as the previous allocation. Formally,

(Individual Rationality from Equal Division) A rule is individual rational from equal division if for each problem $(N, \succeq, t)$ for which $N$ is an admissible coalition,

$$
f_{i}(N, \succeq, t) \succeq_{i} l_{i}+\min \left\{\alpha, u_{i}-l_{i}\right\}
$$


for all $i \in N$, where $\alpha \in \mathbb{R}$ satisfies $\sum_{j \in N} \min \left\{\alpha, u_{j}-l_{j}\right\}=t-\sum_{j \in N} l_{j}{ }^{7}$

The next two properties refer explicitly on how the rule chooses the admissible coalition.

A rule satisfies admissible contraction if the following requirement holds. Consider two problems where the set of admissible coalitions of the first one is contained in the set of admissible coalitions of the second one. Assume that the coalition chosen by the rule in the second problem is admissible for the first one. Then, the rule chooses the same coalition in the two problems.

(Admissible Contraction) A rule satisfies admissible contraction if for any two problems $(N, \succeq, t)$ and $\left(N^{\prime}, \succeq^{\prime}, t^{\prime}\right)$ such that $A C\left(N^{\prime}, \succeq^{\prime}, t^{\prime}\right) \subset A C(N, \succeq, t)$ and $c^{f}(N, \succeq, t) \in$ $A C\left(N^{\prime}, \succeq^{\prime}, t^{\prime}\right)$ then,

$$
c^{f}\left(N^{\prime}, \succeq^{\prime}, t^{\prime}\right)=c^{f}(N, \succeq, t) .
$$

An order $\sigma$ is a one-to-one mapping $\sigma: \mathbb{N} \longrightarrow \mathbb{N}$. A rule satisfies order preservation with respect to $\sigma$ if agent $i$ has more rights to be in the coalition sharing $t$ than any agent that goes after him according to $\sigma$. Namely,

(Order Preservation) A rule satisfies order preservation with respect to $\sigma$ if for each problem $(N, \succeq, t)$ such that $i \notin c^{f}(N, \succeq, t)$ and $c^{f}(N, \succeq, t) \cap\{j \in N \mid \sigma(j)>\sigma(i)\} \neq \varnothing$ then, there is no admissible coalition containing $(\{i\} \cup\{j \in N \mid \sigma(j)<\sigma(i)\}) \cap c^{f}(N, \succeq, t)$.

\subsection{Some basic incompatibilities}

The constant rule assigning zero to all agents in all problems is strategy-proof and anonymous, but it is very inefficient. Proposition 1 below shows that strategy-proofness is a very strong requirement when agents' participation is voluntary. The reason is that the rule has to depend not only on the agents' peaks but also on their intervals of acceptable shares; this makes it too vulnerable to manipulation. Thus, strategy-proof rules do not satisfy other basic and desirable properties related to efficiency.

Proposition 1 The following statements hold:

(1.1) There is no strategy-proof and weak efficient rule.

(1.2) There is no strategy-proof, anonymous and reasonable rule.

Proof To prove (1.1) let $N=\{1,2\}$ be the set of agents, $t=10$ and consider any two profiles $\succeq=\left(\succeq_{1}, \succeq_{2}\right)$ and $\succeq^{\prime}=\left(\succeq_{1}^{\prime}, \succeq_{2}^{\prime}\right)$ characterized by $\left(l_{i}, p_{i}, u_{i}\right)=(4,6,10)$ for $i=1,2$, $\left(l_{1}^{\prime}, p_{1}^{\prime}, u_{1}^{\prime}\right)=(6,8,10)$, and $\left(l_{2}^{\prime}, p_{2}^{\prime}, u_{2}^{\prime}\right)=(6,9,10)$. By weak efficiency, $f\left(\{1,2\},\left(\succeq_{1}, \succeq_{2}^{\prime}\right), 10\right)=$

\footnotetext{
${ }^{7}$ Note that in the division problem with compulsory participation our version of the principle says that $f_{i}(N, \succeq, t) \succeq_{i} \frac{t}{n}$ for all $i \in N$.
} 
$(4,6)$ and $f\left(\{1,2\},\left(\succeq_{1}^{\prime}, \succeq_{2}\right), 10\right)=(6,4)$. By strategy-proofness, $f\left(\{1,2\},\left(\succeq_{1}, \succeq_{2}\right), 10\right)=$ $(4,6)$ to avoid that agent 2 manipulates $f$ at profile $\left(\succeq_{1}, \succeq_{2}^{\prime}\right)$ via $\succeq_{2}$, and $f\left(\{1,2\},\left(\succeq_{1}, \succeq_{2}\right), 10\right)=$ $(6,4)$ to avoid that agent 1 manipulates $f$ at profile $\left(\succeq_{1}^{\prime}, \succeq_{2}\right)$ via $\succeq_{1}$. But this is a contradiction.

To prove (1.2) let $N=\{1,2\}$ be the set of agents, $t=10$ and consider any two profiles $\succeq=\left(\succeq_{1}, \succeq_{2}\right)$ and $\succeq^{\prime}=\left(\succeq_{1}^{\prime}, \succeq_{2}^{\prime}\right)$ characterized by $\left(l_{i}, p_{i}, u_{i}\right)=(2,6,9)$ for $i=1,2$, $\left(l_{1}^{\prime}, p_{1}^{\prime}, u_{1}^{\prime}\right)=(0,3,6)$, and $\left(l_{2}^{\prime}, p_{2}^{\prime}, u_{2}^{\prime}\right)=(6,7,9)$. By anonymity, $f\left(\{1,2\},\left(\succeq_{1}, \succeq_{2}\right), 10\right)$ is equal to either $(0,0)$ or $(5,5)$. Assume $f\left(\{1,2\},\left(\succeq_{1}, \succeq_{2}\right), 10\right)=(0,0)$. Consider any preference $\succeq_{1}^{\prime \prime}$ characterized by $\left(l_{1}^{\prime \prime}, p_{1}^{\prime \prime}, u_{1}^{\prime \prime}\right)=(0.5,4,7.9)$. By reasonability, $f\left(\{1,2\},\left(\succeq_{1}^{\prime \prime}\right.\right.$ ,$\left.\left.\succeq_{2}\right), 10\right)=(4,6)$. Hence, by (P.3), $4 \succ_{1} 0$ and agent 1 manipulates $f$ at $\left(\succeq_{1}, \succeq_{2}\right)$ via $\succeq_{1}^{\prime \prime}$, a contradiction with strategy-proofness. Thus, assume $f\left(\{1,2\},\left(\succeq_{1}, \succeq_{2}\right), 10\right)=(5,5)$ and suppose additionally that $\succeq_{2}$ is such that $5 \sim_{2}$ 8.5. Then, by strategy-proofness, $f_{2}\left(\{1,2\},\left(\succeq_{1}, \succeq_{2}^{\prime}\right), 10\right) \notin(5,8.5)$. By voluntary participation, $f_{2}\left(\{1,2\},\left(\succeq_{1}, \succeq_{2}^{\prime}\right), 10\right)=0$ because otherwise, $f_{2}\left(\{1,2\},\left(\succeq_{1}, \succeq_{2}^{\prime}\right), 10\right) \in[6,9]$ would imply $8.5 \leq f_{2}\left(\{1,2\},\left(\succeq_{1}, \succeq_{2}^{\prime}\right.\right.$ )$, 10) \leq 9$, violating, together with feasibility, voluntary participation of agent 1 at profile $\left(\succeq_{1}, \succeq_{2}^{\prime}\right)$. Thus, $f\left(\{1,2\},\left(\succeq_{1}, \succeq_{2}^{\prime}\right), 10\right)=(0,0)$. Moreover, by reasonability, $f\left(\{1,2\},\left(\succeq_{1}^{\prime}\right.\right.$ ,$\left.\left.\succeq_{2}^{\prime}\right), 10\right)=(3,7)$. But then, by (P.3), $3 \succ_{1} 0$ and agent 1 manipulates $f$ at profile $\left(\succeq_{1}, \succeq_{2}^{\prime}\right)$ via $\succeq_{1}^{\prime}$.

Observe that efficiency is also incompatible with anonymity. To see that, let $N=\{1,2\}$ be the set of agents, $t=1$ and consider any profile $\succeq$ where each $\succeq_{i}$ is characterized by $\left(l_{i}, p_{i}, u_{i}\right)=(1,1,1)$ for $i=1,2$. By anonymity, for all $i \in N, f_{i}(N, \succeq, 1)=k$ for some $k$. By feasibility, $k=0$. But this contradicts efficiency because the feasible allocation $y=(1,0)$ Pareto dominates $(0,0)$.

\section{The uniform rule and some of its extensions}

The uniform rule (Sprumont, 1991) has played a central role in the division problem with compulsory participation because it is the unique rule satisfying different sets of desirable properties. For instance, Sprumont (1991) shows that the uniform rule is the unique rule satisfying strategy-proofness, efficiency and anonymity.

The uniform rule $U$ is defined as follows: for each division problem $(N, \succeq, t)$ and for each $i \in N$,

$$
U_{i}(N, \succeq, t)= \begin{cases}\min \left\{\beta, p_{i}\right\} & \text { if } \sum_{j \in N} p_{j} \geq t \\ \max \left\{\beta, p_{i}\right\} & \text { if } \sum_{j \in N} p_{j}<t\end{cases}
$$

where $\beta$ is the unique number satisfying $\sum_{j \in N} U_{j}(N, \succeq, t)=t$. Namely, $U$ tries to allocate 
the good as equally as possible, keeping the efficient constraints binding: if $\sum_{j \in N} p_{j} \geq t$ then $U_{i}(N, \succeq, t) \leq p_{i}$ for all $i \in N$, and if $\sum_{j \in N} p_{j}<t$ then $U_{i}(N, \succeq, t) \geq p_{i}$ for all $i \in N$.

Observe that when applied to division problems with voluntary participation $U$ is not a rule since at some problems it chooses non-feasible allocations. In the rest of this section we extend the uniform rule to our environment. We do it in two steps. First, we extend the uniform rule only to the subclass of problems where the grand coalition is admissible and the lower bounds of agents' intervals of acceptable shares are equal to zero. Let $(N, \succeq, t)$ be a problem with the properties that $N \in A C(N, \succeq, t)$ and $l_{i}=0$ for all $i \in N$. Then, define $F$ at $(N, \succeq, t)$ as follows: for all $i \in N$,

$$
F_{i}(N, \succeq, t)= \begin{cases}\min \left\{\beta, p_{i}\right\} & \text { if } \sum_{j \in N} p_{j} \geq t \\ \min \left\{\max \left\{\beta, p_{i}\right\}, u_{i}\right\} & \text { if } \sum_{j \in N} p_{j}<t\end{cases}
$$

where $\beta$ is the unique number satisfying $\sum_{j \in N} F_{j}(N, \succeq, t)=t$. Notice that when $\sum_{j \in N} p_{j} \geq$ $t$ (the upper bounds of the participation intervals do not play any role) $F$ coincides with the uniform rule. When $\sum_{j \in N} p_{j}<t$ some of the upper bounds may be binding, so $F$ makes sure that, for all $i \in N, \max \left\{\beta, p_{i}\right\}$ is never larger than $u_{i}$.

But $F$ is not a rule itself because it only applies to a subclass of problems. To define a rule $f$ that extends the egalitarian principle behind the uniform rule (by keeping the bounds imposed by efficiency), select for each problem $(N, \succeq, t)$ an admissible coalition. If the empty set is the unique admissible coalition at $(N, \succeq, t)$, set $f_{i}(N, \succeq, t)=0$ for all $i \in N$. Otherwise, let $c^{f}(N, \succeq, t)$ be the (non-empty) admissible coalition (chosen by $f$ ) among whom $t$ is allocated in two steps. ${ }^{8}$ First, preliminarily assign to each agent in the chosen coalition $c^{f}(N, \succeq, t)$ the lower bound of his interval of acceptable shares, and then apply the rule $F$ to the adjusted problem where the set of agents is $c^{f}(N, \succeq, t)$ and their preferences are $0-$ normalized. Formally, let $(N, \succeq, t)$ be a problem and let $S$ be one of its non-empty admissible coalitions. The adjusted problem $\left(S,\left(\succeq_{j}^{l}\right)_{j \in S}, t-\sum_{j \in S} l_{j}\right)$ is the problem where $S$ is the set of agents, and for each $i \in S, \succeq_{i}^{l}$ is characterized by the triple $\left(0, p_{i}-l_{i}, u_{i}-l_{i}\right)$ and given any pair $x_{i}, y_{i} \in\left[0, u_{i}-l_{i}\right], x_{i} \succeq_{i}^{l} y_{i}$ if and only if $x_{i}+l_{i} \succeq_{i} y_{i}+l_{i}$; i.e., $\succeq_{i}^{l}$ translates $\succeq_{i}$ by substracting $l_{i}$.

(Extended Uniform Rule) We say that $f$ is an extended uniform rule if for all $(N, \succeq$ $, t) \in \mathcal{P}$ and all $i \in N, f_{i}(N, \succeq, t)=0$ whenever $A C(N, \succeq, t)=\{\varnothing\}$ and otherwise,

$f_{i}(N, \succeq, t)= \begin{cases}l_{i}+F_{i}\left(c^{f}(N, \succeq, t),\left(\succeq_{j}^{l}\right)_{j \in c^{f}(N, \succeq, t)}, t-\sum_{j \in c^{f}(N, \succeq, t)} l_{j}\right) & \text { if } i \in c^{f}(N, \succeq, t) \\ 0 & \text { if } i \notin c^{f}(N, \succeq, t)\end{cases}$

\footnotetext{
${ }^{8}$ Remember that for a given problem there may be many admissible coalitions; hence, to fully describe the rule $f$ we will have to specify how $c^{f}(N, \succeq, t)$ is chosen by $f$. But we will deal with this selection later on.
} 
where $c^{f}(N, \succeq, t) \in A C(N, \succeq, t)$ and $c^{f}(N, \succeq, t) \neq \varnothing$.

Observe again that there are many problems with more than one admissible coalition and hence, there are many extended uniform rules. We exhibit an example of a rule in this family by describing a procedure to select, for each problem, an admissible coalition. This procedure is based on the idea of selecting the admissible coalition by given priority to agents according to a fixed order $\sigma$.

To roughly describe the procedure assume momentarily that $N=\{1, \ldots, n\}$ and $\sigma(i)=i$ for all $i \in N$. If the empty coalition is the unique admissible coalition at $(N, \succeq, t)$ then, choose the empty coalition and the share assigned to each agent is equal to zero. If there are non-empty admissible coalitions at $(N, \succeq, t)$ preselect first those coalitions containing agent 1 ; if there are several, keep only those containing also agent 2 , and so on. If there are no admissible coalitions containing agent 1 , preselect those coalitions containing agent 2 ; if there are several, keep only those containing also agent 3 , and so on.

The formal definition is recursive and depends on the one-to-one mapping $\sigma: \mathbb{N} \longrightarrow \mathbb{N}$. Given $N \in \mathcal{N}$ and $1 \leq k \leq n$ let (abusing a bit the notation) $\sigma^{-1}(k) \equiv i$ be the agent in $N$ such that $|\{j \in N \mid \sigma(j) \leq \sigma(i)\}|=k$; namely, $\sigma^{-1}(1)$ is the agent that goes first according to the order $\sigma$, and in general, for $1 \leq k \leq n, \sigma^{-1}(k)$ is the agent that has exactly $k-1$ agents before him according to $\sigma$. Thus, given $\sigma$, we define the extended uniform rule $F^{\sigma}$ as follows. If $A C(N, \succeq, t)=\{\varnothing\}$ then set $F_{i}^{\sigma}(N, \succeq, t)=0$ for all $i \in N$. Assume now that the set of admissible coalitions $A C(N, \succeq, t)$ for problem $(N, \succeq, t)$ contains at least one non-empty coalition.

- Stage 0 (initialization): Given $A C(N, \succeq, t)$, set $X^{0} \equiv A C(N, \succeq, t)$ and go to Stage 1.

- Stage 1 (definition of $X^{1}$ ): Given $X^{0}$, the output of Stage 0.

1. If for each $S \in X^{0}, \sigma^{-1}(1) \notin S$ then, set $X^{1} \equiv X^{0}$ and go to Stage 2 .

2. If there exists $S \in X^{0}$ such that $\sigma^{-1}(1) \in S$ then, set $X^{1} \equiv\left\{S \in X^{0} \mid \sigma^{-1}(1) \in S\right\}$ and go to Stage 2.

- Stage $k$ (definition of $X^{k}$ ): Given $X^{k-1}$, the output of Stage $k-1$.

1. If for each $S \in X^{k-1}, \sigma^{-1}(k) \notin S$ then, set $X^{k} \equiv X^{k-1}$ and go to Stage $k+1$.

2. If there exists $S \in X^{k-1}$ such that $\sigma^{-1}(k) \in S$ then, set $X^{k} \equiv\left\{S \in X^{k-1} \mid \sigma^{-1}(k) \in S\right\}$ and go to Stage $k+1$. 
The procedure stops at Stage $n$ with $X^{n} \equiv X^{n}(N, \succeq, t)$ having a unique coalition. Observe that $X^{n}(N, \succeq, t) \in A C(N, \succeq, t)$. Then, the $\sigma$-extended uniform rule $F^{\sigma}$ is the extended uniform rule such that, for each $(N, \succeq, t) \in \mathcal{P}, F_{i}^{\sigma}(N, \succeq, t)=0$ for all $i \in N$ whenever $A C(N, \succeq, t)=\{\varnothing\}$ and $c^{F^{\sigma}}(N, \succeq, t)=X^{n}(N, \succeq, t)$ otherwise.

\section{Results}

We are now ready to describe and state the main results of the paper. They axiomatically identify three nested subclasses of extended uniform rules. All of them use the same principle to allocate the amount of the good (the same one used by the uniform rule for division problems with compulsory participation) but differ on how to select the admissible coalition. The larger class imposes only two restrictions on the choice of the admissible coalition. First, it chooses the full set of agents whenever it is admissible. Second, it chooses the coalition coherently. The three axioms characterizing this class are efficiency, consistency and individual rationality from equal division. The intermediate class consists of those extended uniform rules that choose the admissible coalition according to a priority relation among all groups of agents that comes from a given monotonic order. This priority ordering on $\mathcal{N}$ has to be monotonic in a double sense. First, adding an agent to a given set gives priority to the larger set. Second, if a set $S$ has priority over a set $T$ then the priority is maintained after adding a player $i \notin S \cup T$ to both sets. This class is identified by the same axioms characterizing the larger class plus the property of admissible contraction. Finally, the smaller class consists of those extended uniform rules that choose the admissible coalition according to an order $\sigma$ on $\mathbb{N}$ that gives priority directly to agents; namely, it is the class of all $\sigma$-extended uniform rules that have been defined in the previous section. This class consists of all efficient, consistent, and individually rational from equal division rules that satisfy order preservation with respect to some $\sigma$. We now turn to the formal statements of the three results.

Theorem 1 characterizes all efficient, consistent, and individually rational from equal division rules as a subclass of extended uniform rules.

Theorem 1 Let $f$ be a rule. Then, $f$ is efficient, consistent, and individually rational from equal division if and only if $f$ is an extended uniform rule with the properties that, for all $(N, \succeq, t) \in \mathcal{P}$,

(1.a) $c^{f}(N, \succeq, t)=N$ when $N$ is an admissible coalition at $(N, \succeq, t)$.

(1.b) $c^{f}\left(S, \succeq_{S}, t-\sum_{i \in N \backslash S} f_{i}(N, \succeq, t)\right)=c^{f}(N, \succeq, t) \cap S$ for each $S \subset N$.

Moreover, the three properties are independent. 


\section{Proof See Appendix 1.}

Theorem 2 characterizes all efficient, consistent, and individually rational from equal division rules that satisfy admissible contraction as the subclass of extended uniform rules with the property that they choose the admissible coalition according to a monotonic order given directly to coalitions (which is not necessarily induced by a unique order of agents). Formally, let $\rho$ be a liner order on $\mathcal{N}$; i.e., $\rho$ is a complete, antisymmetric and transitive binary relation on $\mathcal{N}$. We say that the order $\rho$ is monotonic if:

(i) for all $S \in \mathcal{N}$ and $i \notin S,(S \cup\{i\}) \rho S$, and

(ii) for all $S, T \in \mathcal{N}$ and $i \notin S \cup T, S \rho T$ implies $(S \cup\{i\}) \rho(T \cup\{i\})$.

Theorem 2 Let $f$ be a rule. Then, $f$ is efficient, consistent, individually rational from equal division and satisfies admissible contraction if and only if $f$ is an extended uniform rule with the property that there exists a monotonic order $\rho$ on $\mathcal{N}$ satisfying the property that for all $(N, \succeq, t) \in \mathcal{P}$, (2.a) $c^{f}(N, \succeq, t) \rho S$ for all $S \in A C(N, \succeq, t) \backslash c^{f}(N, \succeq, t)$.

Moreover, the four properties are independent.

Proof See Appendix 2.

Theorem 3 characterizes, for each order $\sigma$ on $\mathbb{N}$, the extended uniform rule $F^{\sigma}$ as the unique efficient, consistent, and individually rational from equal division rule that satisfies order preservation with respect to $\sigma$.

Theorem 3 Let $f$ be a rule and let $\sigma$ be an order. Then, $f$ is efficient, consistent, individually rational from equal division and satisfies order preservation with respect to $\sigma$ if and only if $f=F^{\sigma}$. Moreover, the four properties are independent.

Proof See Appendix 3.

\section{Final remarks}

Before moving to the proofs of the three theorems, few remarks are in order.

First, the (larger) class of extended uniform rules identified in Theorem 1 satisfy also several appealing properties.

A rule satisfies the property of independence of irrelevant agents if at a given problem an agent receives the zero share then, at the problem where the agent is not present anymore, all other agents receive the same share they had received in the original problem. Formally, 
(Independence of Irrelevant Agents) A rule $f$ is independent of irrelevant agents if for each problem $\left(N, \succeq_{N}, t\right)$ such that $f_{i}\left(N, \succeq_{N}, t\right)=0$ for some agent $i \in N$ then, $f_{j}\left(N, \succeq_{N}, t\right)=f_{j}\left(N \backslash\{i\}, \succeq_{N \backslash\{i\}}, t\right)$ for all $j \in N \backslash\{i\}$.

A rule satisfies non-bossiness if one agent receives the same share at two problems that are identical except for the preferences of this agent then, the shares of all the other agents also coincide at the two problems. Formally,

(NON-BOSSY) A rule $f$ is non-bossy if for each problem $(N, \succeq, t)$, each agent $i \in N$, and each $i^{\prime}$ s preferences $\succeq_{i}^{\prime}$ such that $f_{i}\left(N,\left(\succeq_{i}, \succeq_{N \backslash\{i\}}\right), t\right)=f_{i}\left(N,\left(\succeq_{i}^{\prime}, \succeq_{N \backslash\{i\}}\right), t\right)$ then, $f_{j}\left(N,\left(\succeq_{i}, \succeq_{N \backslash\{i\}}\right), t\right)=f_{j}\left(N,\left(\succeq_{i}^{\prime}, \succeq_{N \backslash\{i\}}\right), t\right)$ for all $j \in N \backslash\{i\}$.

A rule satisfies maximality if the set of agents that receive a positive share constitutes (according to set-wise inclusion) a maximal admissible coalition.

(Maximality) A rule is maximal if the following holds. Let $S$ be an admissible coalition for the problem $(N, \succeq, t)$ and assume that $\sum_{j \in S} f_{j}(N, \succeq, t)=t$ and $f_{i}(N, \succeq, t)<l_{i}$ for all $i \in N \backslash S$. Then, for any $T \supsetneq S, T$ is not an admissible coalition for $(N, \succeq, t)$.

By condition (1.a) in Theorem 1, all efficient, consistent and individually rational from equal division rules are maximal. Moreover, Remark 1 below states that non-bossyness and independence of irrelevant agents follow from consistency.

Remark 1 Let $f$ be a consistent rule. Then, $f$ is independent of irrelevant agents and non-bossy.

To show that the statement in Remark 1 holds, assume $f$ is consistent. It follows immediately that $f$ is independent of irrelevant agents. To show that $f$ is non-bossy, consider a problem $\left(N, \succeq_{N}, t\right)$, an agent $i \in N$ and a preference $\succeq_{i}^{\prime}$ such that

$$
f_{i}\left(N,\left(\succeq_{i}, \succeq_{N \backslash\{i\}}\right), t\right)=f_{i}\left(N,\left(\succeq_{i}^{\prime}, \succeq_{N \backslash\{i\}}\right), t\right) .
$$

Since $f$ is consistent, for all $j \in N \backslash\{i\}$,

$$
\begin{aligned}
& f_{j}\left(N,\left(\succeq_{i}, \succeq_{N \backslash\{i\}}\right), t\right)=f_{j}\left(N \backslash\{i\}, \succeq_{N \backslash\{i\}}, t-f_{i}\left(N,\left(\succeq_{i}, \succeq_{N \backslash\{i\}}\right), t\right)\right) \text { and } \\
& f_{j}\left(N,\left(\succeq_{i}^{\prime}, \succeq_{N \backslash\{i\}}\right), t\right)=f_{j}\left(N \backslash\{i\}, \succeq_{N \backslash\{i\}}, t-f_{i}\left(N,\left(\succeq_{i}^{\prime}, \succeq_{N \backslash\{i\}}\right), t\right)\right) .
\end{aligned}
$$

By (1), $f_{j}\left(N,\left(\succeq_{i}, \succeq_{N \backslash\{i\}}\right), t\right)=f_{j}\left(N,\left(\succeq_{i}^{\prime}, \succeq_{N \backslash\{i\}}\right), t\right)$. Hence, $f$ is non-bossy.

Second, there are several natural extensions of our model that we do not consider here but we are planning to study in other papers. First, the existence of voluntary participation constraints suggests as special interesting case the subdomain of preferences where the lower bounds of agents' intervals of acceptable shares are zero (we already used 
this subdomain in the definition of extended uniform rules). Second, the incompatibility of strategy-proofness with the most basic efficiency properties suggests also the interest of studying the set of allocations that are obtained as outcomes of Nash equilibria in direct revelation games induced by rules satisfying desirable properties like those characterized in our results (for instance, the $\sigma$-extended uniform rule).

Third, in some steps in the proofs of the theorems we use profiles $\succeq$ where agents' intervals of acceptable shares depend on a small number $\varepsilon>0$ and are degenerated since for all $i \in N, l_{i}=p_{i}=u_{i}$. However, we could also choose $\varepsilon>0$ in such a way that for each $i \in N, \succeq_{i}$ could be characterized by $\left(l_{i}, p_{i}, u_{i}\right)$ with $0<l_{i}<p_{i}<u_{i}$. However, the case $l_{i}=p_{i}=u_{i}$ makes the arguments more transparent.

\section{References}

[1] Barberà, S. "Notes on Strategy-proof Social Choice Functions," in Social Choice Reexaminated, Arrow, K., A. Sen, A., and K. Suzumura editors, MacMillan (1996). French version: "Sur les fonctions de choix non manipulables," Revue d'Économie Politique 106, 61-81 (1996).

[2] Barberà, S. "An Introduction to Strategy-proof Social Choice Functions," Social Choice and Welfare 18, 619-653 (2001).

[3] Barberà, S., M. Jackson, and A. Neme. "Strategy-proof Allotment Rules," Games and Economic Behavior 18, 1-21 (1997).

[4] Ching, S. "A Simple Characterization of the Uniform Rule," Economics Letters 40, 57-60 (1992).

[5] Ching, S. "An Alternative Characterization of the Uniform Rule," Social Choice and Welfare 11, 131-136 (1994).

[6] Ching, S. and S. Serizawa. "A Maximal Domain for the Existence of Strategy-proof Rules," Journal of Economic Theory 78, 157-166 (1998).

[7] Dagan, N. "A Note on Thomson's Characterizations of the Uniform Rule," Journal of Economic Theory 69, 255-261 (1996).

[8] Jackson, M. "A Crash Course in Implementation Theory," Social Choice and Welfare 18, 655-708 (2001). 
[9] Schummer, J. and W. Thomson. "Two Derivations of the Uniform Rule and an Application to Bankruptcy," Economics Letters 55, 333-337 (1997).

[10] Sprumont, Y. "The Division Problem with Single-peaked Preferences: A Characterization of the Uniform Allocation Rule," Econometrica 59, 509-519 (1991).

[11] Sprumont, Y. "Strategy-proof Collective Choice in Economic and Political Environments," Canadian Journal of Economics 28, 68-107 (1995).

[12] Sönmez, T. "Consistency, Monotonicity, and the Uniform Rule," Economics Letters 46: 229-235 (1994).

[13] Thomson, W. "Consistent Solutions to the Problem of Fair Division when Preferences are Single-peaked," Journal of Economic Theory 63, 219-245 (1994).

[14] Thomson, W. "Population Monotonic Solutions to the Problem of Fair Division when Preferences are Single-peaked," Economic Theory 5, 229-246 (1995).

[15] Thomson, W. "The Replacement Principle in Economies with Single-peaked Preferences," Journal of Economic Theory 76, 145-168 (1997).

[16] Weymark, J. "Sprumont's Characterization of the Uniform Rule when all Singlepeaked Preferences are Admissible," Review of Economic Design 4, 389-393 (1999). 


\section{Appendix 1. Proof of Theorem 1}

\section{A1.1. Preliminaries}

We first introduce the property of bilateral peaks-and-bounds onlyness. It says that for problems with only two agents at which the set of the two agents is an admissible coalition, the rule depends only on the peaks and the bounds of the two agents' preferences.

(Bilateral Peaks-And-Bounds Only) A rule $f$ is bilateral peaks-and-bounds only if for any pair of problems $(N, \succeq, t)$ and $\left(N, \succeq^{\prime}, t\right)$ with $|N|=2, N \in A C(N, \succeq, t)$, and $\left(l_{i}, p_{i}, u_{i}\right)=\left(l_{i}^{\prime}, p_{i}^{\prime}, u_{i}^{\prime}\right)$ for each $i \in N$, then $f(N, \succeq, t)=f\left(N, \succeq^{\prime}, t\right)$.

Before proving Theorem 1 we state and prove two claims and one lemma. The proofs of the two claims adapt to our setting the corresponding proofs of Lemmata 5 and 6 in Dagan (1996).

CLAIM 1 Let $f$ be an efficient and consistent rule that satisfies individual rationality from equal division. Then, $f$ satisfies bilateral peaks-and-bounds onlyness.

Proof of Claim 1 Let $(N, \succeq, t),\left(N^{*}, \succeq^{*}, t\right) \in \mathcal{P}$ be such that $N=\{i, j\}, N^{*}=\{k, m\}$, $\{i, j\} \cap\{k, m\}=\varnothing, \succeq_{i}=\succeq_{k}^{*}, \succeq_{j}=\succeq_{m}^{*}, N \in A C(N, \succeq, t)$, and $N^{*} \in A C\left(N^{*}, \succeq^{*}, t\right)$. Define $x=f\left(N \cup N^{*},\left(\succeq, \succeq^{*}\right), 2 t\right)$. Since $N$ and $N^{*}$ are admissible at their respective problems, $N \cup N^{*} \in A C\left(N \cup N^{*},\left(\succeq, \succeq^{*}\right), 2 t\right)$. By (P.3), efficiency implies $x_{i}+x_{j}+x_{k}+x_{m}=2 t$.

Since $f$ is consistent,

$$
f_{i}\left(\{i,, k\},\left(\succeq_{i}, \succeq_{k}^{*}\right), x_{i}+x_{k}\right)=x_{i} \text { and } f_{k}\left(\{i, k\},\left(\succeq_{i}, \succeq_{k}^{*}\right), x_{i}+x_{k}\right)=x_{k}
$$

Since $f$ satisfies individual rationality from equal division,

$$
f_{i}\left(\{i, k\},\left(\succeq_{i}, \succeq_{k}^{*}\right), x_{i}+x_{k}\right)=f_{k}\left(\{i, k\},\left(\succeq_{i}, \succeq_{k}^{*}\right), x_{i}+x_{k}\right)
$$

Thus, $x_{i}=x_{k}$. Similarly, we conclude that $x_{j}=x_{m}$. Thus, $x_{i}+x_{j}=x_{k}+x_{m}=t$. By consistency,

$$
f_{i}(N, \succeq, t)=x_{i}=x_{k}=f_{k}\left(N^{*}, \succeq^{*}, t\right) \text { and } f_{j}(N, \succeq, t)=x_{j}=x_{m}=f_{m}\left(N^{*}, \succeq^{*}, t\right)
$$

Now, let $\succeq^{\prime}=\left(\succeq_{i}^{\prime}, \succeq_{j}^{\prime}\right)$ be such that $\left(l_{i}^{\prime}, p_{i}^{\prime}, u_{i}^{\prime}\right)=\left(l_{i}, p_{i}, u_{i}\right)$ and $\left(l_{j}^{\prime}, p_{j}^{\prime}, u_{j}^{\prime}\right)=\left(l_{j}, p_{j}, u_{j}\right)$. We want to show that $f(N, \succeq, t)=f\left(N, \succeq^{\prime}, t\right)$. Define $x^{\prime}=f\left(N \cup N^{*},\left(\succeq^{\prime}, \succeq^{*}\right), 2 t\right)$. Using arguments similar to those used above we can conclude that

$$
\begin{aligned}
& f_{i}\left(N, \succeq^{\prime}, t\right)=x_{i}^{\prime}=x_{k}^{\prime}=f_{k}\left(N^{*}, \succeq^{*}, t\right) \text { and } \\
& f_{j}\left(N, \succeq^{\prime}, t\right)=x_{j}^{\prime}=x_{m}^{\prime}=f_{m}\left(N^{*}, \succeq^{*}, t\right) .
\end{aligned}
$$


Thus, $f(N, \succeq, t)=f\left(N, \succeq^{\prime}, t\right)$.

Claim 2 Let $f$ be an efficient and consistent rule that satisfies individual rationality from equal division and let $(\{i, j\}, \succeq, t) \in \mathcal{P}$ be such that $\{i, j\}$ is an admissible coalition. Then, $f_{k}(\{i, j\}, \succeq, t)=l_{k}+F_{k}\left(\{i, j\}, \succeq^{l}, t-l_{i}-l_{j}\right)$ for all $k \in\{i, j\}$.

Proof of Claim 2 Let $(\{i, j\}, \succeq, t) \in \mathcal{P}$ be such that $\{i, j\} \in A C(\{i, j\}, \succeq, t)$. For each $k \in\{i, j\}$, define $x_{k}=l_{k}+\min \left\{\alpha, u_{k}-l_{k}\right\}$, where $\alpha \in \mathbb{R}$ is such that $x_{i}+x_{j}=t$ (as in the definition of individual rationality from equal division applied to the problem $(\{i, j\}, \succeq, t))$. We distinguish between the two rationing situations.

Consider the case $p_{i}+p_{j} \leq t$. Assume first that $x_{k} \geq p_{k}$ for all $k \in\{i, j\}$. Since $f$ is efficient and satisfies individual rationality from equal division,

$$
f_{k}(\{i, j\}, \succeq, t)=x_{k}=l_{k}+F_{k}\left(\{i, j\}, \succeq^{l}, t-l_{i}-l_{j}\right)
$$

for all $k \in\{i, j\}$. Without loss of generality assume now that $x_{i}<p_{i}$. Thus, $t-x_{i}=x_{j}>p_{j}$. By efficiency, $f_{i}(\{i, j\}, \succeq, t) \geq p_{i}>x_{i}$. Suppose that $f_{i}(N, \succeq, t)>p_{i}$. We can find $\succeq_{i}^{\prime}$ such that $\left(l_{i}^{\prime}, p_{i}^{\prime}, u_{i}^{\prime}\right)=\left(l_{i}, p_{i}, u_{i}\right)$ and $x_{i} \succ_{i}^{\prime} f_{i}(N, \succeq, t)$. By Claim $1, f_{i}(\{i, j\}, \succeq, t)=$ $f_{i}\left(\{i, j\},\left(\succeq_{i}^{\prime}, \succeq_{j}\right), t\right)$. Let $x_{i}^{\prime}=l_{i}^{\prime}+\min \left\{\alpha^{\prime}, u_{i}^{\prime}-l_{i}^{\prime}\right\}$ be as in the definition of individual rationality from equal division as applied to the problem $\left(\{i, j\},\left(\succeq_{i}^{\prime}, \succeq_{j}\right), t\right)$. It is obvious that $x_{i}^{\prime}=x_{i}$. Hence, $x_{i}^{\prime} \succ_{i}^{\prime} f_{i}\left(\{i, j\},\left(\succeq_{i}^{\prime}, \succeq_{j}\right), t\right)$, which contradicts that $f$ satisfies individual rationality from equal division at the problem $\left(\{i, j\},\left(\succeq_{i}^{\prime}, \succeq_{j}\right), t\right)$. Then, $f_{i}(\{i, j\}, \succeq, t)=$ $p_{i}=l_{i}+F_{i}\left(\{i, j\}, \succeq^{l}, t-l_{i}-l_{j}\right)$ and hence, $f_{j}(\{i, j\}, \succeq, t)=l_{j}+F_{j}\left(\{i, j\}, \succeq^{l}, t-l_{i}-l_{j}\right)$.

A similar argument can be used to show that the desirable statement also holds when $p_{i}+p_{j}>t$.

Lemma 1 Let $f$ be an efficient and consistent rule that satisfies individual rationality from equal division. Let $(N, \succeq, t)$ be a problem at which $N$ is an admissible coalition. Then, for each $i \in N, f_{i}(N, \succeq, t)=l_{i}+F_{i}\left(N, \succeq^{l}, t-\sum_{j \in N} l_{j}\right)$.

Proof of Lemma 1 Let $(N, \succeq, t)$ be an arbitrary problem with $N \in A C(N, \succeq, t)$. We proceed by induction on $|N|$. If $|N|=2$, the result follows from Claim 2. Assume $|N|>2$ and suppose that the statement holds for all problems $\left(N^{\prime}, \succeq^{\prime}, t^{\prime}\right)$ with $\left|N^{\prime}\right|<|N|$ and $N^{\prime} \in A C\left(N^{\prime}, \succeq^{\prime}, t^{\prime}\right)$. We prove that it also holds for $(N, \succeq, t)$. For each $i \in N$, define

$$
g_{i}(N, \succeq, t)=l_{i}+F_{i}\left(N, \succeq^{l}, t-\sum_{j \in N} l_{j}\right) .
$$

Since $N$ is admissible, by individual rationality from equal division, $\sum_{j \in N} f_{j}(N, \succeq, t)=t$. To obtain a contradiction, suppose that $f(N, \succeq, t) \neq g(N, \succeq, t)$. Then, there exist $i, j \in N$ such that

$$
f_{i}(N, \succeq, t)>g_{i}(N, \succeq, t) \text { and } f_{j}(N, \succeq, t)<g_{j}(N, \succeq, t)
$$


Without loss of generality, assume that $i=1$ and $j=2$. Since $f$ is consistent,

$$
\begin{aligned}
& f_{i}(N, \succeq, t)=f_{i}\left(N \backslash\{1\}, \succeq_{N \backslash\{1\}}, t-f_{1}(N, \succeq, t)\right) \text { for all } i \in N \backslash\{1\}, \text { and } \\
& f_{k}(N, \succeq, t)=f_{k}\left(N \backslash\{2\}, \succeq_{N \backslash\{2\}}, t-f_{2}(N, \succeq, t)\right) \text { for all } k \in N \backslash\{2\} .
\end{aligned}
$$

In Claim 4 in the proof of Theorem 1 below we will show (without using this result) that any extended uniform rule is consistent. Thus,

$$
\begin{aligned}
& g_{i}(N, \succeq, t)=g_{i}\left(N \backslash\{1\}, \succeq_{N \backslash\{1\}}, t-g_{1}(N, \succeq, t)\right) \text { for all } i \in N \backslash\{1\}, \text { and } \\
& g_{k}(N, \succeq, t)=g_{k}\left(N \backslash\{2\}, \succeq_{N \backslash\{2\}}, t-g_{2}(N, \succeq, t)\right) \text { for all } k \in N \backslash\{2\} .
\end{aligned}
$$

By the induction hypothesis, for all $i \in N \backslash\{1\}$,

$$
f_{i}\left(N \backslash\{1\}, \succeq_{N \backslash\{1\}}, t-f_{1}(N, \succeq, t)\right)=g_{i}\left(N \backslash\{1\}, \succeq_{N \backslash\{1\}}, t-f_{1}(N, \succeq, t)\right) .
$$

Since $t-f_{1}(N, \succeq, t)<t-g_{1}(N, \succeq, t)$, the definition of $g$ implies that for all $i \in N \backslash\{1\}$,

$$
g_{i}\left(N \backslash\{1\}, \succeq_{N \backslash\{1\}}, t-f_{1}(N, \succeq, t)\right) \leq g_{i}\left(N \backslash\{1\}, \succeq_{N \backslash\{1\}}, t-g_{1}(N, \succeq, t)\right) .
$$

Hence, by (4), (5), (6), and (7), $f_{i}(N, \succeq, t) \leq g_{i}(N, \succeq, t)$ for all $i \in N \backslash\{1\}$. Analogously, $f_{k}(N, \succeq, t) \geq g_{k}(N, \succeq, t)$ for all $k \in N \backslash\{2\}$. Thus, $f_{i}(N, \succeq, t)=g_{i}(N, \succeq, t)$ for all $i \in$ $N \backslash\{1,2\}$. Since $f$ and $g$ are consistent, for each $i \in\{1,2\}$,

$$
\begin{aligned}
& f_{i}(N, \succeq, t)=f_{i}\left(N \backslash\{1,2\}, \succeq_{N \backslash\{1,2\}}, t-\sum_{j \in\{1,2\}} f_{j}(N, \succeq, t)\right), \text { and } \\
& g_{i}(N, \succeq, t)=g_{i}\left(N \backslash\{1,2\}, \succeq_{N \backslash\{1,2\}}, t-\sum_{j \in\{1,2\}} g_{j}(N, \succeq, t)\right) .
\end{aligned}
$$

By the induction hypothesis, $f_{i}(N, \succeq, t)=g_{i}(N, \succeq, t)$ for all $i \in\{1,2\}$, a contradiction with (3).

\section{A1.2. Proof of the characterization}

$(\Longrightarrow)$ Let $f$ be an efficient and consistent rule that satisfies individual rationality from equal division. We first show that $f$ is an extended uniform rule. Let $(N, \succeq, t)$ be an arbitrary problem. By consistency, for each $i \in c^{f}(N, \succeq, t)$,

$$
f_{i}(N, \succeq, t)=f_{i}\left(c^{f}(N, \succeq, t), \succeq_{c^{f}(N, \succeq, t)}, t\right) .
$$

Since $c^{f}(N, \succeq, t)$ is admissible at $\left(c^{f}(N, \succeq, t), \succeq_{c^{f}(N, \succeq, t)}, t\right)$ and $f$ is efficient, consistent and satisfies individual rationality from equal division we deduce, from Lemma 1 , that for all $i \in c^{f}(N, \succeq, t)$,

$$
f_{i}\left(c^{f}(N, \succeq, t), \succeq_{c^{f}(N, \succeq, t)}, t\right)=l_{i}+F_{i}\left(c^{f}(N, \succeq, t), \succeq_{c^{f}(N, \succeq, t)}^{l}, t-\sum_{j \in c^{f}(N, \succeq, t)} l_{j}\right) .
$$


Hence, by (8), $f_{i}(N, \succeq, t)=l_{i}+F_{i}\left(c^{f}(N, \succeq, t), \succeq_{c^{f}(N, \succeq, t)}^{l}, t-\sum_{j \in c^{f}(N, \succeq, t)} l_{j}\right)$. Moreover, for each $i \notin c^{f}(N, \succeq, t), f_{i}(N, \succeq, t)=0$. Thus, $f$ is an extended uniform rule.

To prove that (1.a) holds, let $(N, \succeq, t)$ be a problem at which $N$ is an admissible coalition and take any $i \in N$. By individual rationality from equal division, $f_{i}\left(N, \succeq_{,} t\right) \succeq_{i}$ $l_{i}+\min \left\{\alpha, u_{i}-l_{i}\right\} \in\left[l_{i}, u_{i}\right]$. By definition of $c^{f}(N, \succeq, t), i \in c^{f}(N, \succeq, t)$. Since $i \in N$ was arbitrary, $c^{f}(N, \succeq, t)=N$. Thus, (1.a) holds.

To prove that (1.b) holds, let $(N, \succeq, t)$ be a problem and consider any agent $i \in N$. Since $f$ is consistent, $f_{j}(N, \succeq, t)=f_{j}\left(S, \succeq_{S}, t-\sum_{i \in N \backslash S} f_{i}(N, \succeq, t)\right)$ for each $j \in S$. Now,

$$
\begin{aligned}
c^{f}\left(S, \succeq_{S}, t-\sum_{i \in N \backslash S} f_{i}(N, \succeq, t)\right) & =\left\{j \in S \mid f_{j}\left(S, \succeq_{S}, t-\sum_{i \in N \backslash S} f_{i}(N, \succeq, t)\right) \in\left[l_{j}, u_{j}\right]\right\} \\
& =\left\{j \in S \mid f_{j}(N, \succeq, t) \in\left[l_{j}, u_{j}\right]\right\} \\
& =c^{f}(N, \succeq, t) \backslash \cap S .
\end{aligned}
$$

Thus, (1.b) holds.

$(\Longleftarrow)$ Assume that $f$ is an extended uniform rule that satisfies (1.a) and (1.b). We want to show that $f$ is efficient, consistent and satisfies individual rationality from equal division. We do it by proving Claims 3 to 7 below.

Claim 3 The rule $F$ is efficient and consistent on the subdomain of problems $(N, \succeq, t)$ where $l_{i}=0$ for all $i \in N$ and $N \in A C(N, \succeq, t)$.

Proof of Claim 3 We first prove that $F(N, \succeq, t)$ is Pareto optimal by distinguishing between the two rationing situations.

Assume first that $\sum_{j \in N} p_{j}<t$. Then, $F_{i}(N, \succeq, t)=\min \left\{\max \left\{\beta, p_{i}\right\}, u_{i}\right\}$ for all $i \in N$. Let $x=\left(x_{i}\right)_{i \in N} \in F A(N, \succeq, t)$ be such that $x_{i} \succeq_{i} F_{i}(N, \succeq, t)$ for all $i \in N$. It is obvious that $\sum_{j \in N} x_{j}=t$. We prove that $x_{i}=F_{i}(N, \succeq, t)$ for all $i \in N$ by distinguishing among three possible cases.

Case 1: $F_{i}(N, \succeq, t)=p_{i}$. Since $x_{i} \succeq_{i} F_{i}(N, \succeq, t), x_{i}=p_{i}$.

Case 2: $F_{i}(N, \succeq, t)=u_{i}$. Since $x_{i} \succeq_{i} F_{i}(N, \succeq, t), x_{i} \leq u_{i}$. Suppose that $x_{i}<u_{i}$. As $\sum_{j \in N} x_{j}=\sum_{j \in N} F_{j}(N, \succeq, t)=t$, there exists $k \in N$ such that $x_{k}>F_{k}(N, \succeq, t)$. By its definition, $F_{k}(N, \succeq, t)$ can only take three different values. If $F_{k}(N, \succeq, t)=u_{k}$ then, $x_{k}>u_{k}$ which contradicts that $x \in F A(N, \succeq, t)$. If $F_{k}(N, \succeq, t)=p_{k}$ then, $x_{k}>p_{k}$ which contradicts that $x_{k} \succeq_{k} F_{k}(N, \succeq, t)$. Finally, if $F_{k}(N, \succeq, t)=\beta$ and $p_{k}<\beta<u_{k}$ then, $\beta<$ $x_{k}$. Since $x \in F A(N, \succeq, t), x_{k} \leq u_{k}$, which contradicts, by (P.2), that $x_{k} \succeq_{k} F_{k}(N, \succeq, t)$. Thus, $x_{i}=u_{i}$.

Case 3: $F_{i}(N, \succeq, t)=\beta$ and $\beta>p_{i}$ (if $\beta=p_{i}$, apply Case 1 above). Since $x_{i} \succeq_{i}$ $F_{i}(N, \succeq, t), x_{i} \leq \beta$ by (P.2). Suppose that $x_{i}<\beta$. As $\sum_{j \in N} x_{j}=\sum_{j \in N} F_{j}(N, \succeq, t)=t$, 
there exists $k \in N$ such that $x_{k}>F_{k}(N, \succeq, t)$. Using arguments similar to those already used in Case 2 we obtain a contradiction. Thus, $x_{i}=\beta$.

A similar argument can be used to show that $F(N, \succeq, t)$ is Pareto optimal when $\sum_{j \in N} p_{j} \geq t\left(\right.$ and $F_{i}(N, \succeq, t)=\min \left\{\beta, p_{i}\right\}$ for all $\left.i \in N\right)$.

To prove that $F$ is consistent, it is sufficient to show that for all $i \in N \backslash\{k\}, F_{i}(N, \succeq, t)=$ $F_{i}\left(N \backslash\{k\}, \succeq_{N \backslash\{k\}}, t-f_{k}(N, \succeq, t)\right)$ for any arbitrary agent $k \in N$. Again, we distinguish between the two rationing situations.

Assume first that $\sum_{j \in N} p_{j}<t$. Then, $F_{i}(N, \succeq, t)=\min \left\{\max \left\{\beta, p_{i}\right\}, u_{i}\right\}$ for all $i \in N$. Thus, $p_{i} \leq F_{i}(N, \succeq, t)$ for all $i \in N$. Let $k \in N$. Then, $\sum_{j \in N \backslash\{k\}} p_{j} \leq$ $\sum_{j \in N \backslash\{k\}} F_{j}(N, \succeq, t)$. We distinguish between two possible cases.

Case 1: $\sum_{j \in N \backslash\{k\}} p_{j}<\sum_{j \in N \backslash\{k\}} F_{j}(N, \succeq, t)=t-F_{k}(N, \succeq, t)$. Since

$$
\sum_{j \in N \backslash\{k\}} \min \left\{\max \left\{\beta, p_{j}\right\}, u_{j}\right\}=t-F_{k}(N, \succeq, t),
$$

and $F_{i}\left(N \backslash\{k\}, \succeq_{N \backslash\{k\}}, t-F_{k}(N, \succeq, t)\right)=\min \left\{\max \left\{\beta^{\prime}, p_{i}\right\}, u_{i}\right\}$ where $\beta^{\prime}$ is the unique number satisfying

$$
\sum_{j \in N \backslash\{k\}} \min \left\{\max \left\{\beta^{\prime}, p_{j}\right\}, u_{j}\right\}=t-F_{k}(N, \succeq, t),
$$

we deduce that $\beta=\beta^{\prime}$ and, for each $i \in N \backslash\{k\}$

$$
F_{i}\left(N \backslash\{k\}, \succeq_{N \backslash\{k\}}, t-F_{k}(N, \succeq, t)\right)=\min \left\{\max \left\{\beta, p_{i}\right\}, u_{i}\right\}=F_{i}(N, \succeq, t) .
$$

Case 2: $\sum_{j \in N \backslash\{k\}} p_{j}=\sum_{j \in N \backslash\{k\}} F_{j}(N, \succeq, t)=t-F_{k}(N, \succeq, t)$. Then, by efficiency of $F$, $F_{i}(N, \succeq, t)=p_{i}$ for all $i \in N \backslash\{k\}$. Moreover, for each $i \in N \backslash\{k\}$,

$$
F_{i}\left(N \backslash\{k\}, \succeq_{N \backslash\{k\}}, t-F_{k}(N, \succeq, t)\right)=\min \left\{\beta, p_{i}\right\},
$$

where $\beta$ is the unique number satisfying

$$
\sum_{j \in N \backslash\{k\}} \min \left\{\beta, p_{j}\right\}=t-F_{k}(N, \succeq, t)=\sum_{j \in N \backslash\{k\}} p_{j} .
$$

Thus, $\beta=\max _{j \in N \backslash\{k\}}\left\{p_{j}\right\}$. Hence, for each $i \in N \backslash\{k\}$,

$$
F_{i}\left(N \backslash\{k\}, \succeq_{N \backslash\{k\}}, t-F_{k}(N, \succeq, t)\right)=p_{i} .
$$

The case $\sum_{j \in N} p_{j} \geqslant t$ is similar and we omit it. This concludes the proof of Claim $3 . \square$ Claim 4 The rule $f$ is consistent.

Proof of Claim 4 Let $(N, \succeq, t) \in \mathcal{P}$ and $S \subsetneq N$. We have to show that for all $i \in S$,

$$
f_{i}(N, \succeq, t)=f_{i}\left(S, \succeq_{S}, t-\sum_{j \in N \backslash S} f_{j}(N, \succeq, t)\right)
$$


It is sufficient to prove that it holds for $|S|=n-1$. Let $k \in N$ and $i \in N \backslash\{k\}$. We distinguish between two cases.

Case 1: $i \notin c^{f}(N, \succeq, t)$. Then, $f_{i}(N, \succeq, t)=0$. By (1.b), $c^{f}\left(N \backslash\{k\}, \succeq_{N \backslash\{k\}}, t-f_{k}(N, \succeq, t)\right)=$ $c^{f}(N, \succeq, t) \backslash\{k\}$. Hence, $i \notin c^{f}\left(N \backslash\{k\}, \succeq_{N \backslash\{k\}}, t-f_{k}(N, \succeq, t)\right)$ and then,

$$
f_{i}\left(N \backslash\{k\}, \succeq_{N \backslash\{k\}}, t-f_{k}(N, \succeq, t)\right)=0=f_{i}(N, \succeq, t) .
$$

Case 2: $i \in c^{f}(N, \succeq, t)$. Then, since by hypothesis $f$ is an extended uniform rule,

$$
f_{i}(N, \succeq, t)=l_{i}+F_{i}\left(c^{f}(N, \succeq, t), \succeq_{c^{f}(N, \succeq, t)}^{l}, t-\sum_{j \in c^{f}(N, \succeq, t)} l_{j}\right) .
$$

By (1.b), $i \in c^{f}\left(N \backslash\{k\}, \succeq_{N \backslash\{k\}}, t-f_{k}(N, \succeq, t)\right)=c^{f}(N, \succeq, t) \backslash\{k\}$. Then,

$$
\begin{gathered}
f_{i}\left(N \backslash\{k\}, \succeq_{N \backslash\{k\}}, t-f_{k}(N, \succeq, t)\right)= \\
=l_{i}+F_{i}\left(c^{f}(N, \succeq, t) \backslash\{k\}, \succeq_{c^{f}(N, \succeq, t) \backslash\{k\}}^{l}, t-f_{k}(N, \succeq, t)-\sum_{j \in c^{f}(N, \succeq, t) \backslash\{k\}} l_{j}\right) .
\end{gathered}
$$

We consider two subcases.

Subcase 2.1: $k \notin c^{f}(N, \succeq, t)$. Then, $f_{k}(N, \succeq, t)=0$. Now,

$$
\begin{gathered}
F_{i}\left(c^{f}(N, \succeq, t) \backslash\{k\}, \succeq_{c^{f}(N, \succeq, t) \backslash\{k\}}^{l}, t-f_{k}(N, \succeq, t)-\sum_{j \in c^{f}(N, \succeq, t) \backslash\{k\}} l_{j}\right)= \\
=F_{i}\left(c^{f}(N, \succeq, t), \succeq_{c^{f}(N, \succeq, t)}^{l}, t-\sum_{j \in c^{f}(N, \succeq, t)} l_{j}\right) .
\end{gathered}
$$

Hence,

$$
f_{i}\left(N \backslash\{k\}, \succeq_{N \backslash\{k\}}, t-f_{k}(N, \succeq, t)\right)=f_{i}(N, \succeq, t) .
$$

Subcase 2.2: $k \in c^{f}(N, \succeq, t)$. By Claim 3 above, $F$ is consistent (on the smaller subdomain). Thus, setting $c^{f} \equiv c^{f}(N, \succeq, t)$,

$F_{i}\left(c^{f}, \succeq_{c^{f}}^{l}, t-\sum_{j \in c^{f}} l_{j}\right)=F_{i}\left(c^{f} \backslash\{k\}, \succeq_{c^{f} \backslash\{k\}}^{l}, t-\sum_{j \in c^{f}} l_{j}-F_{k}\left(c^{f}, \succeq_{c^{f}}^{l}, t-\sum_{j \in c^{f}} l_{j}\right)\right)$.

Since $k \in c^{f}$ and $f$ is an extended uniform rule, $-l_{k}-F_{k}\left(c^{f}, \succeq_{c^{f}}^{l}, t-\sum_{j \in c^{f}} l_{j}\right)=$ $-f_{k}(N, \succeq, t)$. Now,

$$
\begin{aligned}
f_{i}(N, \succeq, t) & =l_{i}+F_{i}\left(c^{f}, \succeq_{c^{f}}^{l}, t-\sum_{j \in c^{f}} l_{j}\right) \\
& =l_{i}+F_{i}\left(c^{f} \backslash\{k\}, \succeq_{c^{f} \backslash\{k\}}^{l}, t-\sum_{j \in c^{f} \backslash\{k\}} l_{j}-f_{k}(N, \succeq, t)\right) \\
& =f_{i}\left(N \backslash\{k\}, \succeq_{N \backslash\{k\}}, t-f_{k}(N, \succeq, t)\right) .
\end{aligned}
$$

This concludes the proof of Claim 4 . 
Claim 5 The rule $f$ satisfies individual rationality from equal division.

Proof of Claim 5 Let $(N, \succeq, t)$ be such that $N$ is an admissible coalition. By (1.a), $c^{f}(N, \succeq, t)=N$. Since $f$ is an extended uniform rule,

$$
f_{i}(N, \succeq, t)=l_{i}+F_{i}\left(N, \succeq_{N}^{l}, t-\sum_{j \in N} l_{j}\right)
$$

for all $i \in N$. We will show that for all $i \in N$,

$$
f_{i}(N, \succeq, t) \succeq_{i} l_{i}+\min \left\{\alpha, u_{i}-l_{i}\right\},
$$

where $\sum_{j \in N} \min \left\{\alpha, u_{j}-l_{j}\right\}=t-\sum_{j \in N} l_{j}$, by distinguishing between the two rationing situations.

Assume first that $\sum_{j \in N} p_{j}<t$. Then, $\sum_{j \in N}\left(p_{j}-l_{j}\right)<t-\sum_{j \in N} l_{j}$. Now, for each $i \in N$,

$$
F_{i}\left(N, \succeq^{l}, t-\sum_{j \in N} l_{j}\right)=\min \left\{\max \left\{\beta, p_{i}-l_{i}\right\}, u_{i}-l_{i}\right\}
$$

where $\beta$ is the unique number satisfying $\sum_{j \in N} \min \left\{\max \left\{\beta, p_{j}-l_{j}\right\}, u_{j}-l_{j}\right\}=t-\sum_{j \in N} l_{j}$. Then, $\alpha \geq \beta$ because

$$
\sum_{j \in N} \min \left\{\max \left\{\alpha, p_{j}-l_{j}\right\}, u_{j}-l_{j}\right\} \geq \sum_{j \in N} \min \left\{\alpha, u_{j}-l_{j}\right\}=t-\sum_{j \in N} l_{j} .
$$

Let $i \in N$. We consider separately the following three cases.

Case 1: $\min \left\{\max \left\{\beta, p_{i}-l_{i}\right\}, u_{i}-l_{i}\right\}=p_{i}-l_{i}$. Then, $f_{i}(N, \succeq, t)=p_{i}$ and $f_{i}\left(N, \succeq_{,}\right) \succsim_{i}$ $l_{i}+\min \left\{\alpha, u_{i}-l_{i}\right\}$.

Case 2: $\min \left\{\max \left\{\beta, p_{i}-l_{i}\right\}, u_{i}-l_{i}\right\}=u_{i}-l_{i}>p_{i}-l_{i}$. Then,

$$
\begin{aligned}
\min \left\{\alpha, u_{i}-l_{i}\right\} & =u_{i}-l_{i} \\
f_{i}(N, \succeq, t) & =l_{i}+\left(u_{i}-l_{i}\right)=u_{i}, \text { and } \\
l_{i}+\min \left\{\alpha, u_{i}-l_{i}\right\} & =u_{i} .
\end{aligned}
$$

Thus, $f_{i}(N, \succeq, t) \sim_{i} l_{i}+\min \left\{\alpha, u_{i}-l_{i}\right\}$.

Case 3: $\min \left\{\max \left\{\beta, p_{i}-l_{i}\right\}, u_{i}-l_{i}\right\}=\beta>p_{i}-l_{i}$. We consider two subcases.

Subcase 3.1: $\alpha \leq u_{i}-l_{i}$. Then,

$$
\begin{aligned}
\min \left\{\alpha, u_{i}-l_{i}\right\} & =\alpha, \\
f_{i}(N, \succeq, t) & =l_{i}+\beta, \text { and } \\
l_{i}+\min \left\{\alpha, u_{i}-l_{i}\right\} & =l_{i}+\alpha .
\end{aligned}
$$

Since $l_{i}+\alpha \geq l_{i}+\beta \geq p_{i}$, by (P.2), $f_{i}(N, \succeq, t) \succeq_{i} l_{i}+\min \left\{\alpha, u_{i}-l_{i}\right\}$. 
Subcase 3.2: $\alpha>u_{i}-l_{i}$. Then,

$$
\begin{aligned}
\min \left\{\alpha, u_{i}-l_{i}\right\} & =u_{i}-l_{i}, \\
f_{i}(N, \succeq, t) & =l_{i}+\beta, \text { and } \\
l_{i}+\min \left\{\alpha, u_{i}-l_{i}\right\} & =l_{i}+u_{i}-l_{i}=u_{i} .
\end{aligned}
$$

Since $p_{i}<l_{i}+\beta=f_{i}(N, \succeq, t) \leq u_{i}$, by (P.2), $f_{i}(N, \succeq, t) \succeq_{i} l_{i}+\min \left\{\alpha, u_{i}-l_{i}\right\}$.

The case $\sum_{j \in N} p_{j} \geq t$ is similar and we omit it.

Claim 6 Let $(N, \succeq, t) \in \mathcal{P}$ be such that $S \in A C(N, \succeq, t)$ for some $S \neq \varnothing$, Then $c^{f}(N, \succeq, t) \neq \varnothing$.

Proof of Claim 6 Suppose not. Let $S \neq \varnothing$ be an admissible coalition at $(N, \succeq, t)$. Since $c^{f}(N, \succeq, t)=\varnothing, f_{i}(N, \succeq, t)=0$ for all $i \in N$. By (1.a), $S \neq N$. Let $i_{1} \in N \backslash S$. By (1.b),

$$
\begin{aligned}
c^{f}\left(N \backslash\left\{i_{1}\right\}, \succeq_{N \backslash\left\{i_{1}\right\}}, t\right) & =c^{f}\left(N \backslash\left\{i_{1}\right\}, \succeq_{N \backslash\left\{i_{1}\right\}}, t-f_{i_{1}}(N, \succeq, t)\right) \\
& =c^{f}(N, \succeq, t) \backslash\left\{i_{1}\right\}=\varnothing .
\end{aligned}
$$

If $S \neq N \backslash\left\{i_{1}\right\}$, let $i_{2} \in N \backslash\left(S \cup\left\{i_{1}\right\}\right)$. By (1.b) again,

$c^{f}\left(N \backslash\left\{i_{1}, i_{2}\right\}, \succeq_{N \backslash\left\{i_{1}, i_{2}\right\}}, t-f_{i_{2}}\left(N \backslash\left\{i_{1}\right\}, \succeq_{N \backslash\left\{i_{1}\right\}}, t\right)\right)=c^{f}\left(N \backslash\left\{i_{1}\right\}, \succeq_{N \backslash\left\{i_{1}\right\}}, t\right) \backslash\left\{i_{2}\right\}=\varnothing$.

By Claim $4, f$ is consistent. Then,

$$
\begin{aligned}
f_{i_{2}}\left(N \backslash\left\{i_{1}\right\}, \succeq_{N \backslash\left\{i_{1}\right\}}, t\right) & =f_{i_{2}}\left(N \backslash\left\{i_{1}\right\}, \succeq_{N \backslash\left\{i_{1}\right\}}, t-f_{i_{1}}(N, \succeq, t)\right) \\
& =f_{i_{2}}(N, \succeq, t)=0 .
\end{aligned}
$$

Thus,

$c^{f}\left(N \backslash\left\{i_{1}, i_{2}\right\}, \succeq_{N \backslash\left\{i_{1}, i_{2}\right\}}, t\right)=c^{f}\left(N \backslash\left\{i_{1}, i_{2}\right\}, \succeq_{N \backslash\left\{i_{1}, i_{2}\right\}}, t-f_{i_{2}}\left(N \backslash\left\{i_{1}\right\}, \succeq_{N \backslash\left\{i_{1}\right\}}, t\right)\right)=\varnothing$.

Iterating this argument, we conclude that $c^{f}\left(S, \succeq_{S}, t\right)=\varnothing$. Since $S$ is admissible at $(N, \succeq, t), S$ is admissible at $\left(S, \succeq_{S}, t\right)$ as well. Moreover, $f$ satisfies $\left(1\right.$. a). Thus, $c^{f}\left(S, \succeq_{S}, t\right)=$ $S$, a contradiction.

Claim 7 The rule $f$ is efficient.

Proof of Claim 7 Suppose not. Then, there exist $(N, \succeq, t) \in \mathcal{P}, x \in F A(N, \succeq, t)$, and $j \in N$ such that $x_{i} \succeq_{i} f_{i}(N, \succeq, t)$ for all $i \in N$ and $x_{j} \succ_{j} f_{j}(N, \succeq, t)$. We denote $c^{x}=\left\{i \in N \mid l_{i} \leq x_{i} \leq u_{i}\right\}$. Since $x_{j} \succ_{j} f_{j}(N, \succeq, t), j \in c^{x}$ and hence $c^{x} \neq \varnothing$. Moreover, 
$c^{f}(N, \succeq, t) \subset c^{x}$. Since $c^{x}$ is an admissible coalition at $(N, \succeq, t)$, by Claim $6, c^{f}(N, \succeq, t) \neq$ $\varnothing$.

Since $f$ satisfies consistency and $c^{f}(N, \succeq, t) \subset c^{x}, f_{i}\left(c^{x}, \succeq_{c^{x}}, t\right)=f_{i}(N, \succeq, t)$ for all $i \in c^{x}$. By $(1 . a), c^{f}\left(c^{x}, \succeq_{c^{x}}, t\right)=c^{x}$. By $(1 . b), c^{f}\left(c^{x}, \succeq_{c^{x}}, t\right)=c^{f}(N, \succeq, t) \cap c^{x}$. Thus, $c^{x}=c^{f}(N, \succeq, t)$. Now $\left(x_{i}-l_{i}\right)_{i \in c^{f}(N, \succeq, t)}$ Pareto dominates

$$
\left(F_{i}\left(c^{f}(N, \succeq, t),\left(\succeq_{j}^{l}\right)_{j \in c^{f}(N, \succeq, t)}, t-\sum_{j \in c^{f}(N, \succeq, t)} l_{j}\right)\right)_{i \in c^{f}(N, \succeq, t)},
$$

which contradicts Claim 3.

This finishes the proof of the characterization in Theorem 1.

\section{A1.3. Independence of the axioms}

Let $\sigma: \mathbb{N} \longrightarrow \mathbb{N}$ be the identity order; i.e., $\sigma(i)=i$ for all $i \in \mathbb{N}$.

Consider the rule $f^{1}$ defined as follows. Given $(N, \succeq, t) \in \mathcal{P}$, set $c^{f^{1}}(N, \succeq, t)=$ $c^{F^{\sigma}}(N, \succeq, t)$ and

$$
f_{i}^{1}(N, \succeq, t)= \begin{cases}0 & \text { if } i \notin c^{f^{1}}(N, \succeq, t) \\ l_{i}+\min \left\{\alpha, u_{i}-l_{i}\right\} & \text { if } i \in c^{f^{1}}(N, \succeq, t),\end{cases}
$$

where $\alpha \in \mathbb{R}$ satisfies $\sum_{j \in c^{1}{ }^{1}(N, \succeq, t)} \min \left\{\alpha, u_{j}-l_{j}\right\}=t-\sum_{j \in c^{f^{1}}(N, \succeq, t)} l_{j}$. It is not difficult to prove that $f^{1}$ is consistent, satisfies individual rationality from equal division, but it is not efficient.

Consider the rule $f^{2}$ defined as follows. Given $(N, \succeq, t) \in \mathcal{P}$, set $c^{f^{2}}(N, \succeq, t)=$ $c^{F^{\sigma}}(N, \succeq, t)$ and

$$
f_{i}^{2}(N, \succeq, t)= \begin{cases}0 & \text { if } i \notin c^{f^{2}}(N, \succeq, t) \\ D_{i}^{\sigma}\left(c^{f^{2}}(N, \succeq, t), \succeq_{c^{f^{2}}(N, \succeq, t)}, t\right) & \text { if } i \in c^{f^{2}}(N, \succeq, t)\end{cases}
$$

where $D_{i}^{\sigma}\left(c^{f^{2}}(N, \succeq, t), \succeq_{c^{f^{2}}(N, \succeq, t)}, t\right)$ denotes the sequential dictatorial rule induced by the order $\sigma$ in the problem $\left(c^{f^{2}}(N, \succeq, t), \succeq_{c^{f^{2}}(N, \succeq, t)}, t\right)$. In the sequential dictatorial rule agents select, following the order $\sigma$, the shares they most prefer, as long as there is enough amount of the good (we skip its formal definition). It is not difficult to prove that $f^{2}$ is efficient, consistent but it is not individually rational from equal division.

Let $\sigma^{\prime}: \mathbb{N} \longrightarrow \mathbb{N}$ be any order different from $\sigma$. Consider the rule $f^{3}$ defined as follows. First, define $f^{1, \sigma^{\prime}}$ similarly to $f^{1}$ but using order $\sigma^{\prime}$ instead of $\sigma$. Now, for all $(N, \succeq, t) \in \mathcal{P}$,

$$
f^{3}(N, \succeq, t)= \begin{cases}f^{1}(N, \succeq, t) & \text { if }|N| \text { is odd } \\ f^{1, \sigma^{\prime}}(N, \succeq, t) & \text { if }|N| \text { is even. }\end{cases}
$$


It is not difficult to prove that $f^{3}$ is efficient, satisfies individual rationality from equal division but it is not consistent.

\section{Appendix 2. Proof of Theorem 2}

\section{A2.1. Proof of the characterization}

$(\Longleftarrow)$ We first prove that if $f$ is an extended uniform rule with the property that there exists a monotonic order $\rho$ on $\mathcal{N}$ such that (2.a) holds then, $f$ is efficient, consistent, individually rational from equal division and satisfies admissible contraction. We do it by proving Claims 8 and 9 below.

Claim 8 The rule $f$ is efficient, consistent and satisfies individual rationality from equal division.

Proof of Claim 8 By Theorem 1, it is sufficient to prove that $f$ satisfies (1.a) and (1.b). We first show that $f$ satisfies (1.a). Let $(N, \succeq, t) \in \mathcal{P}$ be such that $N$ is admissible and let $\rho$ be the monotonic order on $\mathcal{N}$ associated to $f$. By property $(i)$ of $\rho, N \rho S$ for all $S \subsetneq N$. Thus, $c^{f}(N, \succeq, t)=N$.

We now prove that $f$ satisfies (1.b). Let $(N, \succeq, t) \in \mathcal{P}$ and $i \in N$. We consider separately the following two cases.

Case 1: $i \notin c^{f}(N, \succeq, t)$. Then, $f_{i}(N, \succeq, t)=0$. Obviously,

$$
c^{f}(N, \succeq, t) \in A C\left(N \backslash\{i\}, \succeq_{N \backslash\{i\}}, t-f_{i}(N, \succeq, t)\right)
$$

and

$$
A C\left(N \backslash\{i\}, \succeq_{N \backslash\{i\}}, t-f_{i}(N, \succeq, t)\right) \subseteq A C(N, \succeq, t) .
$$

By (2.a), $c^{f}(N, \succeq, t) \rho S$ for all $S \in A C\left(N \backslash\{i\}, \succeq_{N \backslash\{i\}}, t-f_{i}(N, \succeq, t)\right) \backslash c^{f}(N, \succeq, t)$, which means that

$$
\begin{aligned}
c^{f}\left(N \backslash\{i\}, \succeq_{N \backslash\{i\}}, t-f_{i}(N, \succeq, t)\right) & =c^{f}(N, \succeq, t) \\
& =c^{f}(N, \succeq, t) \backslash\{i\} .
\end{aligned}
$$

Case 2: $i \in c^{f}(N, \succeq, t)$. Then, $f_{i}(N, \succeq, t) \in\left[l_{i}, u_{i}\right]$. It is easy to see that

$$
S \in A C\left(N \backslash\{i\}, \succeq_{N \backslash\{i\}}, t-f_{i}(N, \succeq, t)\right) \text { implies } S \cup\{i\} \in A C(N, \succeq, t) .
$$

Moreover, $c^{f}(N, \succeq, t) \backslash\{i\} \in A C\left(N \backslash\{i\}, \succeq_{N \backslash\{i\}}, t-f_{i}(N, \succeq, t)\right)$ holds. We prove that $\left(c^{f}(N, \succeq, t) \backslash\{i\}\right) \rho S$ for all $S \in A C\left(N \backslash\{i\}, \succeq_{N \backslash\{i\}}, t-f_{i}(N, \succeq, t)\right) \backslash\left(c^{f}(N, \succeq, t) \backslash\{i\}\right)$. 
Suppose not; there exists $S^{\prime} \in A\left(N \backslash\{i\}, \succeq_{N \backslash\{i\}}, t-f_{i}(N, \succeq, t)\right)$ such that $S^{\prime} \rho\left(c^{f}(N, \succeq, t) \backslash\{i\}\right)$. By (9), $S^{\prime} \cup\{i\} \in A(N, \succeq, t)$. By property (ii) of $\rho,\left(S^{\prime} \cup\{i\}\right) \rho c^{f}(N, \succeq, t)$, which contra$\operatorname{dicts}(2 . \mathrm{a})$.

Claim 9 The rule $f$ satisfies admissible contraction.

Proof of Claim 9 Let $(N, \succeq, t)$ and $\left(N^{\prime}, \succeq^{\prime}, t^{\prime}\right)$ be any two problems with the property that $A C\left(N^{\prime}, \succeq^{\prime}, t^{\prime}\right) \subseteq A C(N, \succeq, t)$ and $c^{f}(N, \succeq, t) \in A C\left(N^{\prime}, \succeq^{\prime}, t^{\prime}\right)$. By (2.a), $c^{f}(N, \succeq, t) \rho S$ for all $S \in A C(N, \succeq, t) \backslash c^{f}(N, \succeq, t)$. Since $A C\left(N^{\prime}, \succeq^{\prime}, t^{\prime}\right) \subseteq A C(N, \succeq, t)$ and $c^{f}(N, \succeq, t) \in A\left(N^{\prime}, \succeq^{\prime}, t^{\prime}\right), c^{f}(N, \succeq, t) \rho S$ for all $S \in A C\left(N^{\prime}, \succeq^{\prime}, t^{\prime}\right) \backslash c^{f}(N, \succeq, t)$. By (2.a), $c^{f}\left(N^{\prime}, \succeq^{\prime}, t^{\prime}\right)=c^{f}(N, \succeq, t)$.

$(\Longrightarrow)$ Let $f$ be an efficient and consistent rule that satisfies individual rationality from equal division and admissible contraction. By Theorem $1, f$ is an extended uniform rule. We want to show that there exists a monotonic order $\rho$ on $\mathcal{N}$ such that $f$ satisfies (2.a).

We first define (using $f$ ) a binary relation $\rho$ on $\mathcal{N}$. Let $S, S^{\prime} \in \mathcal{N}$. Three cases are possible.

Case 1: $S \supset S^{\prime}$. Then, set $S \rho S^{\prime}$.

Case 2: $S^{\prime} \supset S$. Then, set $S^{\prime} \rho S$.

Case 3: There exist agents $j \in S \backslash S^{\prime}$ and $j^{\prime} \in S^{\prime} \backslash S$. Consider any problem $(N, \succeq, t)$ where $S, S^{\prime} \subseteq N$ and for each $i \in N, l_{i}=p_{i}=u_{i}$, and

$$
p_{i}= \begin{cases}\varepsilon & \text { if } i \in S \cap S^{\prime} \\ \varepsilon^{2} & \text { if } i \in S \backslash\left(S^{\prime} \cup\{j\}\right) \\ t-\varepsilon\left|S \cap S^{\prime}\right|-\varepsilon^{2}\left|S \backslash\left(S^{\prime} \cup\{j\}\right)\right| & \text { if } i=j \\ \varepsilon^{3} & \text { if } i \in S^{\prime} \backslash\left(S \cup\left\{j^{\prime}\right\}\right) \\ t-\varepsilon\left|S \cap S^{\prime}\right|-\varepsilon^{3}\left|S^{\prime} \backslash\left(S \cup\left\{j^{\prime}\right\}\right)\right| & \text { if } i=j^{\prime} \\ \varepsilon^{4} & \text { if } i \in N \backslash\left(S \cup S^{\prime}\right) .\end{cases}
$$

Moreover, we choose $\varepsilon>0$ small enough to make sure that $0<p_{i}<t$ for all $i \in N$ and $A C(N, \succeq, t)=\left\{S, S^{\prime}\right\}$. Observe that such $\varepsilon>0$ exists. Since $f$ is efficient, $c^{f}(N, \succeq, t) \in$ $\left\{S, S^{\prime}\right\}$. Then, if $c^{f}(N, \succeq, t)=S$ set $S \rho S^{\prime}$ and if $c^{f}(N, \succeq, t)=S^{\prime}$ set $S^{\prime} \rho S$.

Since $f$ satisfies admissible contraction, $\rho$ does not depend on $(N, \succeq, t)$. Namely, let $\left(N^{\prime}, \succeq^{\prime}, t^{\prime}\right)$ be such that $A C\left(N^{\prime}, \succeq^{\prime}, t^{\prime}\right)=\left\{S, S^{\prime}\right\}$. Then, $c^{f}\left(N^{\prime}, \succeq^{\prime}, t^{\prime}\right)=c^{f}(N, \succeq, t)$. Thus, $\rho$ is well defined.

Claim 10 If $S \rho S^{\prime}$ and $T \subset S \cap S^{\prime}$ then, $(S \backslash T) \rho\left(S^{\prime} \backslash T\right)$.

Proof of Claim 10 If $S \supset S^{\prime}$ then, the statement follows immediately. Assume $S \backslash S^{\prime} \neq \varnothing$ and $S^{\prime} \backslash S \neq \varnothing$ hold. Let $i \in T \subset S \cap S^{\prime}$ and $(N, \succeq, t)$ be a problem as in the 
definition of $\rho$ applied to $S$ and $S^{\prime}$. Thus, $A C(N, \succeq, t)=\left\{S, S^{\prime}\right\}, c^{f}(N, \succeq, t)=S$ and $A C\left(N \backslash\{i\}, \succeq_{N \backslash\{i\}}, t-f_{i}(N, \succeq, t)\right)=\left\{S \backslash\{i\}, S^{\prime} \backslash\{i\}\right\}$. Since $f$ satisfies (1.b),

$$
c^{f}\left(N \backslash\{i\}, \succeq_{N \backslash\{i\}}, t-f_{i}(N, \succeq, t)\right)=c^{f}(N, \succeq, t) \backslash\{i\}=S \backslash\{i\} .
$$

Let $\left(N, \succeq^{\prime}, t\right)$ be as in the definition of $\rho$ applied to $S \backslash\{i\}$ and $S^{\prime} \backslash\{i\}$. Thus,

$$
A C\left(N, \succeq^{\prime}, t\right)=\left\{S \backslash\{i\}, S^{\prime} \backslash\{i\}\right\}=A C\left(N \backslash\{i\}, \succeq_{N \backslash\{i\}}, t-f_{i}(N, \succeq, t)\right) .
$$

Since $f$ satisfies admissible contraction and $c^{f}\left(N \backslash\{i\}, \succeq_{N \backslash\{i\}}, t-f_{i}(N, \succeq, t)\right)=S \backslash\{i\}$, $c^{f}\left(N, \succeq^{\prime}, t\right)=S \backslash\{i\}$. Thus, $(S \backslash\{i\}) \rho\left(S^{\prime} \backslash\{i\}\right)$. Repeating successively the same argument for each agent in $T \backslash\{i\}$ it follows that $(S \backslash T) \rho\left(S^{\prime} \backslash T\right)$.

Claim 11 The binary relation $\rho$ on $\mathcal{N}$ is complete, antisymmetric, and satisfies properties $(i)$ and $(i i)$.

Proof of Claim 11 By definition, $\rho$ is a complete and antisymmetric binary relation. Property $(i)$ holds trivially. Suppose that $\rho$ does not satisfy property $(i i)$. Then, there exist $S, T \subset N$ and $i \in N \backslash(S \cup T)$ such that $S \rho T$ but $(S \cup\{i\}) \rho(T \cup\{i\})$ does not hold. Since $\rho$ is complete, $(T \cup\{i\}) \rho(S \cup\{i\})$. By Claim 10,T $\rho$, which is a contradiction.

Lemma 2 The rule $f$ satisfies (2.a).

Proof of Lemma 2 Let $S \in A C(N, \succeq, t) \backslash c^{f}(N, \succeq, t)$. We want to prove that $c^{f}(N, \succeq, t) \rho S$. We distinguish among the following three cases.

Case 1: $S \subsetneq c^{f}(N, \succeq, t)$. Then $c^{f}(N, \succeq, t) \rho S$ by definition of $\rho$.

Case 2: $c^{f}(N, \succeq, t) \subsetneq S$. We will obtain a contradiction. Consider the problem $\left(S, \succeq_{S}, t\right)$. Since $S \in A C(N, \succeq, t), S \in A C\left(S, \succeq_{S}, t\right)$. By Theorem 1, $f$ satisfies (1.a). Thus, $c^{f}\left(S, \succeq_{S}, t\right)=S$. Since $c^{f}(N, \succeq, t) \subsetneq S, c^{f}(N, \succeq, t) \in A C\left(S, \succeq_{S}, t\right)$. Moreover, $A C\left(S, \succeq_{S}, t\right) \subseteq$ $A C(N, \succeq, t)$. Since $f$ satisfies admissible contraction, $c^{f}\left(S, \succeq_{S}, t\right)=c^{f}(N, \succeq, t)$, a contradiction with $c^{f}\left(S, \succeq_{S}, t\right)=S$.

Case 3: $c^{f}(N, \succeq, t) \backslash S \neq \varnothing$ and $S \backslash c^{f}(N, \succeq, t) \neq \varnothing$. Let $\left(N, \succeq^{\prime}, t^{\prime}\right)$ be as in the definition of $\rho$ applied to the sets $c^{f}(N, \succeq, t)$ and $S$. Thus, $A C\left(N, \succeq^{\prime}, t^{\prime}\right)=\left\{c^{f}(N, \succeq, t), S\right\}$. Since $f$ satisfies admissible contraction, $c^{f}\left(N, \succeq^{\prime}, t^{\prime}\right)=c^{f}(N, \succeq, t)$. By the definition of $\rho$, $c^{f}(N, \succeq, t) \rho S$.

Lemma 3 below states that $\rho$ is transitive, the only remaining property to be proven in order to finish the proof of the characterization of Theorem 2.

Lemma 3 The binary relation $\rho$ on $\mathcal{N}$ is transitive.

Proof of Lemma 3 To simplify the notation, given a family $\left\{X_{1}, X_{2}, \ldots, X_{K}\right\}$ of subsets of $N$, we denote $\cup_{k=1}^{K} X_{k}$ by $X_{1} X_{2} \ldots X_{K}$. Assume that $S \rho S^{\prime}$ and $S^{\prime} \rho S^{\prime \prime}$. We must prove that 
$S \rho S^{\prime \prime}$. We decompose $S, S^{\prime}$, and $S^{\prime \prime}$ according to Figure 1, with $S=A B C G, S^{\prime}=C D E G$ and $S^{\prime \prime}=A E F G$, and prove Claims 12-16 below.

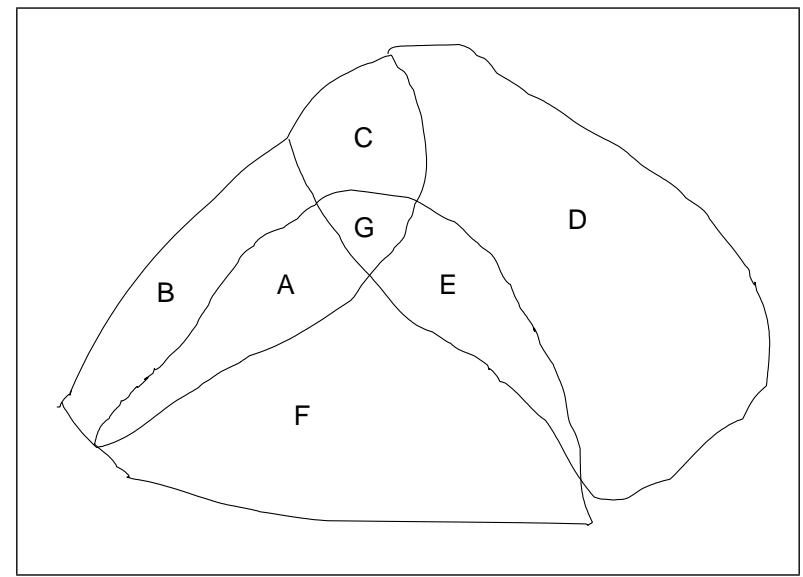

Figure 1

Claim 12 Assume that $A C(N, \succeq, t)=\left\{X_{k}\right\}_{k=1}^{K}$ and for each $k \neq 1$, there exists $j_{k}$ such that $X_{j_{k}} \rho X_{k}$. Then, $X_{1} \rho X_{k}$ for each $k \neq 1$.

Proof of Claim 12 Since $f$ is efficient, $c^{f}(N, \succeq, t) \in A C(N, \succeq, t)$. Let $k \neq 1$ and assume $X_{j_{k}} \rho X_{k}$. Since $f$ satisfies $(2 . \mathrm{a}), c^{f}(N, \succeq, t) \neq X_{k}$. Thus, $c^{f}(N, \succeq, t)=X_{1}$. Since $f$ satisfies (2.a), $X_{1} \rho X_{k}$ for each $k \neq 1$.

Claim 13 Assume that $B \neq \varnothing, D \neq \varnothing$, and $F \neq \varnothing$. Then, $S \rho S^{\prime \prime}$.

Proof of Claim 13 By assumption, for each $X \in\{B, D, F\}$, we can find $i_{X} \in X$. Consider any problem $(N, \succeq, 1)$ where $B D F \subseteq N$ and for all $i \in N, l_{i}=p_{i}=u_{i}$ and

$$
p_{i}= \begin{cases}\varepsilon & \text { if } i \in G \\ \varepsilon^{2} & \text { if } i \in C \\ \varepsilon^{3} & \text { if } i \in A \\ \varepsilon^{4} & \text { if } i \in E \\ \varepsilon^{5} & \text { if } i \in B \backslash\left\{i_{B}\right\} \\ 1-\varepsilon|G|-\varepsilon^{2}|C|-\varepsilon^{3}|A|-\varepsilon^{5}\left|B \backslash\left\{i_{B}\right\}\right| & \text { if } i=i_{B} \\ \varepsilon^{6} & \text { if } i \in D \backslash\left\{i_{D}\right\} \\ 1-\varepsilon|G|-\varepsilon^{2}|C|-\varepsilon^{4}|E|-\varepsilon^{6}\left|D \backslash\left\{i_{D}\right\}\right| & \text { if } i=i_{D} \\ \varepsilon^{7} & \text { if } i \in F \backslash\left\{i_{F}\right\} \\ 1-\varepsilon|G|-\varepsilon^{2}|C|-\varepsilon^{4}|E|-\varepsilon^{7}\left|F \backslash\left\{i_{F}\right\}\right| & \text { if } i=i_{F} \\ 2 & \text { otherwise. }\end{cases}
$$


For $\varepsilon>0$ sufficiently small, $A C(N, \succeq, 1)=\left\{S, S^{\prime}, S^{\prime \prime}\right\}$. By Claim $12, c^{f}(N, \succeq, 1)=S$. Since $f$ satisfies (2.a), $S \rho S^{\prime \prime}$.

Claim 14 Let $U, U^{\prime}, V, V^{\prime}$ be such that $X \cap Y=\varnothing$ for each $X, Y \in\left\{U, U^{\prime}, V, V^{\prime}\right\}$ with $X \neq Y$ and assume $U \rho U^{\prime}$ and $V \rho V^{\prime}$. Then, $U V \rho U^{\prime} V^{\prime}$.

Proof of Claim 14 Since $U \rho U^{\prime}$ and $V \rho V^{\prime}, U \neq \varnothing$ and $V \neq \varnothing$ hold. We consider four cases separately.

Case 1: $U^{\prime}=V^{\prime}=\varnothing$. Obviously, $U V \rho \varnothing$.

Case 2: $U^{\prime} \neq \varnothing$ and $V^{\prime} \neq \varnothing$. For each $X \in\left\{U, U^{\prime}, V, V^{\prime}\right\}$, take $i_{X} \in X$. Consider any problem $(N, \succeq, 3)$ where $U U^{\prime} V V^{\prime} \subseteq N$, and for all $i \in N, l_{i}=p_{i}=u_{i}$ and

$$
p_{i}= \begin{cases}\varepsilon & \text { if } i \in U \backslash\left\{i_{U}\right\} \\ 2-\varepsilon\left|U \backslash\left\{i_{U}\right\}\right| & \text { if } i=i_{U} \\ \varepsilon^{2} & \text { if } i \in U^{\prime} \backslash\left\{i_{U^{\prime}}\right\} \\ 2-\varepsilon^{2}\left|U^{\prime} \backslash\left\{i_{U^{\prime}}\right\}\right| & \text { if } i=i_{U^{\prime}} \\ \varepsilon^{3} & \text { if } i \in V \backslash\left\{i_{V}\right\} \\ 1-\varepsilon^{3}\left|V \backslash\left\{i_{V}\right\}\right| & \text { if } i=i_{V} \\ \varepsilon^{4} & \text { if } i \in V^{\prime} \backslash\left\{i_{V^{\prime}}\right\} \\ 1-\varepsilon^{4}\left|V^{\prime} \backslash\left\{i_{V^{\prime}}\right\}\right| & \text { if } i=i_{V^{\prime}} \\ 4 & \text { otherwise. }\end{cases}
$$

It is easy to see that, for $\varepsilon>0$ is sufficiently small, $A C(N, \succeq, 3)=\left\{U V, U V^{\prime}, U^{\prime} V, U^{\prime} V^{\prime}\right\}$. Since $U \rho U^{\prime}$ and, by Claim 11, $\rho$ satisfies property $(i i), U V \rho U^{\prime} V$ and $U V^{\prime} \rho U^{\prime} V^{\prime}$. Since $V \rho V^{\prime}$, and again by property (ii), $U V \rho U V^{\prime}$. Claim 12 implies $U V \rho U^{\prime} V^{\prime}$.

Case 3: $U^{\prime} \neq \varnothing$ and $V^{\prime}=\varnothing$. For each $X \in\left\{U, U^{\prime}, V\right\}$, take $i_{X} \in X$. Consider any problem $(N, \succeq, 1)$ where $U U^{\prime} V \subseteq N$ for all $i \in N \backslash\left\{i_{U}\right\}, l_{i}=p_{i}=u_{i}$, and for $\varepsilon>0$ small enough,

$$
p_{i}= \begin{cases}\varepsilon & \text { if } i \in U \backslash\left\{i_{U}\right\} \\ \varepsilon^{2} & \text { if } i \in U^{\prime} \backslash\left\{i_{U^{\prime}}\right\} \\ 1-\varepsilon^{2}\left|U^{\prime} \backslash\left\{i_{U^{\prime}}\right\}\right| & \text { if } i=i_{U^{\prime}} \\ \varepsilon^{3} & \text { if } i \in V \\ 4 & \text { otherwise, }\end{cases}
$$

and $l_{i_{U}}=1-\varepsilon\left|U \backslash\left\{i_{U}\right\}\right|-\varepsilon^{3}|V|$ and $u_{i_{U}}=1-\varepsilon\left|U \backslash\left\{i_{U}\right\}\right|$. Now, $A C(N, \succeq, 1)=U^{\prime} \cup$ $\{X \mid U \subset X \subset U V\}$. Since $U \rho U^{\prime}$ and $U V \rho X$ for each $X \in A C(N, \succeq, 1) \backslash\left\{U V, U^{\prime}\right\}$, by Claim 12, $U V \rho U^{\prime}$.

Case 4: $U^{\prime}=\varnothing$ and $V^{\prime} \neq \varnothing$. Since the argument is symmetric to the previous case, we omit it. 
Claim 15 Let $U, V$ be such that $U \cap V=\varnothing$ and $U \rho V$. Then, for each $X \subset V, U \rho X$.

Proof of Claim 15 If $X=\varnothing$, then $U \rho X$ follows from property $(i)$ of $\rho$. Assume $X \neq \varnothing$ and take $i_{X} \in X$ and $i_{U} \in U$. Consider any problem $(N, \succeq, 1)$ with $U V \subseteq N$ and for all $i \in N \backslash\left\{i_{X}\right\}, l_{i}=p_{i}=u_{i}$ and for $\varepsilon>0$ small enough,

$$
p_{i}= \begin{cases}\varepsilon & \text { if } i \in U \backslash\left\{i_{U}\right\} \\ 1-\varepsilon\left|U \backslash\left\{i_{U}\right\}\right| & \text { if } i=i_{U} \\ \varepsilon^{2} & \text { if } i \in X \backslash\left\{i_{X}\right\} \\ \varepsilon^{3} & \text { if } i \in V \backslash X \\ 4 & \text { otherwise, }\end{cases}
$$

and $l_{i_{X}}=1-\varepsilon^{2}\left|X \backslash\left\{i_{X}\right\}\right|-\varepsilon^{3}|V \backslash X|$ and $u_{i_{X}}=1-\varepsilon^{2}\left|X \backslash\left\{i_{X}\right\}\right|$. Now $A C(N, \succeq, 1)=$ $U \cup\{Y \mid X \subset Y \subset V\}$. Since $U \rho V$ and $V \rho Y$ for each $Y \in A(N, \succeq, 1) \backslash\{V, U\}$ we conclude, by Claim 12, that $U \rho X$.

Claim 16 Assume that for each $X, Y \in\{A, B, C, D, E, F\}, X \cap Y=\varnothing, A B \rho D E$, and $C D \rho A F$. Then, $A B C D \rho D E A F$.

Proof of Claim 16 We first prove that if $B \neq \varnothing, D \neq \varnothing$, and $F \neq \varnothing$, then $A B C D \rho D E A F$. Let $S=A B C, S^{\prime}=C D E$, and $S^{\prime \prime}=A E F$. Since $A B \rho D E, C D \rho A F$, and $\rho$ satisfies property $(i i), S=A B C \rho C D E=S^{\prime}$ and $S^{\prime}=C D E \rho A E F=S^{\prime \prime}$. By Claim 13, $S=A B C \rho A E F=S^{\prime \prime}$. By Claim 10, BC $\rho E F$. By property $(i i)$ of $\rho, A B C D \rho D E A F$.

We now prove that if $C \neq \varnothing, A \neq \varnothing$, and $E \neq \varnothing$, then $A B C D \rho D E A F$. Let $S=B C D$, $S^{\prime}=A B F$, and $S^{\prime \prime}=D E F$. Since $A B \rho D E, C D \rho A F$, and $\rho$ satisfies property (ii), $S^{\prime}=$ $A B F \rho D E F=S^{\prime \prime}$ and $S=B \rho A B F=S^{\prime}$. By Claim 13, $S=C D B \rho D E F=S^{\prime \prime}$. By Claim 10, $B C \rho E F$. By property (ii) of $\rho, A B C D \rho D E A F$.

We proceed by considering several cases:

Case 1: $A=\varnothing, D=\varnothing$. Thus, $B \rho E$ and $C \rho F$. Then, $B C \rho E F$ follows from Claim 14 and hence $A B C D \rho D E A F$.

Case 2: $A=\varnothing, D \neq \varnothing$. Thus, $B \rho D E$ and $C D \rho F$. Since $B \rho D E, B \neq \varnothing$. We consider two subcases.

Subcase 2.1: $F \neq \varnothing$. Since $B \neq \varnothing, D \neq \varnothing$, and $F \neq \varnothing, A B C D \rho D E A F$ holds.

Subcase 2.2: $F=\varnothing$. Thus, $B \rho D E$ and $C \rho D$. By property $(i i)$ of $\rho$, it is sufficient to to prove that $B C \rho E$. Since $B \rho D E$, by Claim $15, B \rho E$. Since $C \rho \varnothing$ and Claim 14 holds, $B C \rho E$. Thus, $A B C D \rho D E A F$.

Case 3: $A \neq \varnothing, D=\varnothing$. It is symmetric to Case 2 .

Case 4: $A \neq \varnothing, D \neq \varnothing$. We consider three subcases. 
Subcase 4.1: $B \neq \varnothing, F \neq \varnothing$. Since $B \neq \varnothing, D \neq \varnothing$, and $F \neq \varnothing, A B C D \rho D E A F$ holds.

Subcase 4.2: $B \neq \varnothing, F=\varnothing$. Thus, $A B \rho D E$ and $C D \rho A$. By property (ii) of $\rho$, it is sufficient to to prove that $B \rho E$. First, if $E=\varnothing$ it holds trivially. Second, assume $E \neq \varnothing$ and $C \neq \varnothing$ hold. Then, and since $C \neq \varnothing, A \neq \varnothing$, and $E \neq \varnothing, A B C D \rho D E A F$ holds. Finally, assume $E \neq \varnothing$ and $C=\varnothing$ hold. Suppose $E \rho B$. By Claim 14, $D E \rho A B$, which contradicts that $A B \rho D E$.

Subcase 4.3: $B=\varnothing$. Thus, $A \rho D E$ and $C D \rho A F$. We first prove that $C \neq \varnothing$. Suppose not. Then, $D \rho A F$. By Claim 15, $D \rho A$. Since $A \rho D E$, and by Claim 15 again, $A \rho D$, which contradicts the antisymmetry of $\rho$. Hence, $C \neq \varnothing$. First, assume $E=\varnothing$. Thus, $A \rho D$ and $C D \rho A F$. By property (ii) of $\rho$, it is sufficient to to prove that $C \rho F$. Suppose not. Then, $F \rho C$. Since $A \rho D$ and Claim 14, $F A \rho C D$, which contradicts that $\rho$ is antisymmetric and $C D \rho A F$. Second, assume $E \neq \varnothing$. Since $C \neq \varnothing, A \neq \varnothing$, and $E \neq \varnothing, A B C D \rho D E A F$ holds.

To conclude with the proof of Lemma 3, assume $S \rho S^{\prime}$ and $S^{\prime} \rho S^{\prime \prime}$. We want to show that $S \rho S^{\prime \prime}$ holds. Since $S \rho S^{\prime}, A B C G \rho C D E G$ (see Figure 1). By Claim 10, $A B \rho D E$. Since $S^{\prime} \rho S^{\prime \prime}, C D E G \rho A E F G$. By Claim 10, $C D \rho A F$. By Claim 16, $A B C D \rho D E A F$. By Claim $10, B C \rho E F$. By property (ii) of $\rho, S=B C A G \rho E F A G=S^{\prime \prime}$.

\section{A2.2. The independence of the axioms}

Let $\sigma$ be such that $\sigma(i)=i$ for all $i \in \mathbb{N}$. Given $S, T \in \mathcal{N}$ define $\mathbf{1}^{S, S \cup T} \in \mathbb{R}^{S \cup T}$ as follows:

$$
\mathbf{1}_{i}^{S, S \cup T}= \begin{cases}1 & \text { if } i \in S \\ 0 & \text { if } i \notin S .\end{cases}
$$

Define $\mathbf{1}^{T, S \cup T}$ analogously. We define the order $\rho$ on $\mathcal{N}$. For any $S, T \in \mathcal{N}, S \neq T$, set $S \rho T$ if and only if $\mathbf{1}^{S, S \cup T}$ is strictly larger, according to the lexicographic order, than $\mathbf{1}^{T, S \cup T}$. Now, it is easy to see that for any problem $(N, \succeq, t), c^{F^{\sigma}}(N, \succeq, t) \in A C(N, \succeq, t)$ and $c^{F^{\sigma}}(N, \succeq, t) \rho S$ for all $S \in A C(N, \succeq, t) \backslash c^{F^{\sigma}}(N, \succeq, t)$. It is not difficult to prove that, as defined in A1.3 of Appendix 1,

(1) $f^{1}$ is consistent, individually rational from equal division and satisfies admissible contraction but it is not efficient;

(2) $f^{2}$ is efficient, consistent and satisfies admissible contraction but it is not individually rational from equal division; and

(3) $f^{3}$ is efficient and individually rational from equal division and satisfies admissible contraction but it is not consistent. 
We define $f^{4}$ as follows. Let $\sigma^{\prime}$ be the order in which agent 1 is always the last and the other agents are ordered as in $\sigma$. Now, for all $(N, \succeq, t) \in \mathcal{P}$,

$$
f^{4}(N, \succeq, t)= \begin{cases}F^{\sigma^{\prime}}(N, \succeq, t) & \text { if } 1 \in N \text { and } p_{1}=1 \\ F^{\sigma}(N, \succeq, t) & \text { otherwise. }\end{cases}
$$

It is not difficult to prove that $f^{4}$ is efficient, consistent, individually rational from equal division but it does not satisfy admissible contraction.

\section{Appendix 3. Proof of Theorem 3}

\section{A3.1. Proof of the characterization}

$(\Longleftarrow)$ Let $\sigma: \mathbb{N} \longrightarrow \mathbb{N}$ be an order. We first prove that the extended uniform rule $F^{\sigma}$ is efficient, consistent, individually rational from equal division and satisfies order preservation with respect to $\sigma$. We do it in Claims 17 and 18 below. In order to simplify the notation, assume $\sigma(i)=i$ for all $i \in \mathbb{N}$.

CLAIM 17 The extended uniform rule $F^{\sigma}$ is efficient, consistent and individually rational from equal division.

Proof of Claim 17 By Theorem 1, it is sufficient to prove that $F^{\sigma}$ satisfies (1.a) and (1.b). By its definition, $F^{\sigma}$ satisfies (1.a). To show that $F^{\sigma}$ also satisfies (1.b), consider any problem $(N, \succeq, t)$ and let $i \in N$ be arbitrary. For each $1 \leq j \leq n-1$, let $X^{\prime j}$ denote the sets $X^{j}$ as in the definition of $F^{\sigma}$ when the procedure is applied to the problem $\left(N \backslash\{i\}, \succeq_{N \backslash\{i\}}, t-F_{i}^{\sigma}(N, \succeq, t)\right)$. We will prove that

$$
c^{F^{\sigma}}(N, \succeq, t) \backslash\{i\} \in X^{\prime j} \text { for all } 1 \leq j \leq n-1 .
$$

Observe that (1.b) would follow because (10) and $\left|X^{\prime n-1}\right|=1$ imply that $c^{F^{\sigma}}(N, \succeq, t) \backslash\{i\}=$ $X^{\prime n-1}$ and hence, $c^{F^{\sigma}}\left(N \backslash\{i\}, \succeq_{N \backslash\{i\}}, t-F_{i}^{\sigma}(N, \succeq, t)\right)=c^{F^{\sigma}}(N, \succeq, t) \backslash\{i\}$. To prove (10) we consider separately two cases.

Case 1: $F_{i}^{\sigma}(N, \succeq, t) \in\left[l_{i}, u_{i}\right]$. Thus, $i \in c^{f}(N, \succeq, t)$. We first mention two statements:

(s1) Let $S \in A C\left(N \backslash\{i\}, \succeq_{N \backslash\{i\}}, t-F_{i}^{\sigma}(N, \succeq, t)\right)$. Then, $\sum_{j \in S} l_{j} \leq t-F_{i}^{\sigma}(N, \succeq, t) \leq$ $\sum_{j \in S} u_{j}$. Hence, $\sum_{j \in S \cup\{i\}} l_{j} \leq t \leq \sum_{j \in S \cup\{i\}} u_{j}$. Namely, $S \cup\{i\} \in A C(N, \succeq, t)$.

(s2) Let $S \in A C(N, \succeq, t)$ be such that $i \in S$ and there exists $\left(x_{j}\right)_{j \in S} \in F A\left(S, \succeq_{S}, t\right)$ such that $x_{i}=F_{i}^{\sigma}(N, \succeq, t)$. Thus, $S \backslash\{i\} \in A C\left(N \backslash\{i\}, \succeq_{N \backslash\{i\}}, t-F_{i}^{\sigma}(N, \succeq, t)\right)$. 
Since $c^{F^{\sigma}}(N, \succeq, t) \in X^{0} \equiv A C(N, \succeq, t)$ and $(s 2)$ holds,

$$
c^{F^{\sigma}}(N, \succeq, t) \backslash\{i\} \in X^{\prime 0}=A C\left(N \backslash\{i\}, \succeq_{N \backslash\{i\}}, t-F_{i}^{\sigma}(N, \succeq, t)\right) .
$$

We now prove that $c^{F^{\sigma}}(N, \succeq, t) \backslash\{i\} \in X^{\prime j}$ for all $1 \leq j \leq n-1$. We do it for $j=1$, the first step of the procedure (the other steps are similar and we omit them). We consider two subcases.

Subcase 1.1: For each $S \in X^{0}, 1 \notin S$. Then $X^{1}=X^{0}$. Suppose that $1 \in S$ for some $S \in X^{\prime 0}$. By $(s 1), S \cup\{i\} \in X^{0}$, which is a contradiction. Then, for each $S \in X^{\prime 0}, 1 \notin S$. Hence $X^{\prime 1}=X^{\prime 0}$ and $c^{F^{\sigma}}(N, \succeq, t) \backslash\{i\} \in X^{\prime 1}$.

Subcase 1.2: There exists $S \in X^{0}$ such that $1 \in S$. Then, $X^{1}=\left\{S \in X^{0} \mid 1 \in S\right\}$. Again, we consider two subcases.

Subcase 1.2.1: $i \neq 1$. Since $c^{F^{\sigma}}(N, \succeq, t) \in X^{1}$, by $(s 2), 1 \in c^{F^{\sigma}}(N, \succeq, t) \backslash\{i\} \in X^{\prime 0}$. Now $X^{\prime 1}=\left\{S \in X^{\prime 0} \mid 1 \in S\right\}$ and hence $c^{F^{\sigma}}(N, \succeq, t) \backslash\{i\} \in X^{\prime 1}$.

Subcase 1.2.2: $i=1$. In this case we can not compute $X^{\prime 1}$. After $X^{\prime 0}$ we must compute $X^{\prime 2}$. We prove that $c^{f}(N, \succeq, t) \backslash\{i\} \in X^{\prime 2}$. We again consider two subcases.

Subcase 1.2.2.1: For each $S \in X^{1}, 2 \notin S$. Then $X^{2}=X^{1}$. Suppose that $2 \in S$ for some $S \in X^{\prime 0}$. By $(s 1), S \cup\{1\} \in X^{0}$, which is a contradiction. Then, for each $S \in X^{\prime 0}, 2 \notin S$. Hence $X^{\prime 2}=X^{\prime 0}$ and $c^{F^{\sigma}}(N, \succeq, t) \backslash\{1\} \in X^{\prime 2}$.

Subcase 1.2.2.2: There exists $S \in X^{1}$ such that $2 \in S$. Then $X^{2}=\left\{S \in X^{2} \mid 2 \in S\right\}$. Since $c^{F^{\sigma}}(N, \succeq, t) \in X^{2}$, by $(s 2), 2 \in c^{F^{\sigma}}(N, \succeq, t) \backslash\{1\} \in X^{\prime 0}$. Now $X^{\prime 2}=\left\{S \in X^{\prime 0} \mid 2 \in S\right\}$ and hence $c^{F^{\sigma}}(N, \succeq, t) \backslash\{1\} \in X^{\prime 2}$.

Case 2: $F_{i}^{\sigma}(N, \succeq, t) \notin\left[l_{i}, u_{i}\right]$. Then, $F_{i}^{\sigma}(N, \succeq, t)=0<l_{i}$ and $i \notin c^{f}(N, \succeq, t)$. It is easy to see that $A C\left(N \backslash\{i\}, \succeq_{N \backslash\{i\}}, t\right)=\{S \in A C(N, \succeq, t) \mid i \notin S\}$. Hence, $c^{F^{\sigma}}(N, \succeq, t) \in X^{\prime 0}$. Using arguments similar to those used in Case 1 , we can prove that $c^{F^{\sigma}}(N, \succeq, t) \in X^{\prime j}$ for all $1 \leq j \leq n-1$.

Claim 18 The extended uniform rule $F^{\sigma}$ satisfies order preservation with respect to $\sigma$.

Proof of Claim 18 Let $i \in N$ be such that $i \notin c^{F^{\sigma}}(N, \succeq, t)$ and $c^{F^{\sigma}}(N, \succeq, t) \cap$ $\{i+1, \ldots, n\} \neq \varnothing$. We must prove that there is no admissible coalition containing $\{1, \ldots, i\} \cap$ $c^{F^{\sigma}}(N, \succeq, t)$. To obtain a contradiction, let $S$ be an admissible coalition containing $\{1, \ldots, i\} \cap$ $c^{F^{\sigma}}(N, \succeq, t)$. Let $j \in N$. If there exists $S^{\prime} \in X^{j-1}$ such that $j \in S^{\prime}$, then $X^{j}=$ $\left\{T \in X^{j-1} \mid j \in T\right\}$. Since, $c^{F^{\sigma}}(N, \succeq, t)=X^{n} \subset X^{j}, j \in c^{F^{\sigma}}(N, \succeq, t)$. Thus, if $j \notin$ $c^{F^{\sigma}}(N, \succeq, t),\left\{T \in X^{j-1} \mid j \in T\right\}=\varnothing$ and $X^{j}=X^{j-1}$. We now prove that $S \in X^{j}$ for all $1 \leq j \leq i$. We prove it by induction. First, $S \in X^{0}$ holds and let $1 \leq j \leq i$. Assume that $S \in X^{j-1}$. We prove that $S \in X^{j}$. We distinguish between two possible cases. 
Case 1: $j \notin c^{F^{\sigma}}(N, \succeq, t)$. Thus, $X^{j}=X^{j-1}$, which means that $S \in X^{j}$.

Case 2: $j \in c^{F^{\sigma}}(N, \succeq, t)$. Thus, $X^{j}=\left\{T \in X^{j-1} \mid j \in T\right\}$ and $S \in X^{j}$ because $\{1, \ldots, i\} \cap$ $c^{F^{\sigma}}(N, \succeq, t) \subset S$.

Thus, $i \in S \in X^{i}$, which means that $i \in c^{F^{\sigma}}(N, \succeq, t)$. But this contradicts the initial assumption that $i \notin c^{F^{\sigma}}(N, \succeq, t)$.

$(\Longrightarrow)$ Let $f$ be an efficient and consistent rule that satisfies individual rationality from equal division and order preservation with respect to $\sigma$. By Theorem $1, f$ is an extended uniform rule. Claim 19 below finishes with the proof of the characterization in Theorem 3 .

Claim 19 Let $(N, \succeq, t)$ be a problem. Then, $c^{f}(N, \succeq, t)=c^{F^{\sigma}}(N, \succeq, t)$.

Proof of Claim 19 By definition of $F^{\sigma}, c^{F^{\sigma}}(N, \succeq, t)=X^{n}$. We now prove that if $f$ satisfies order preservation with respect to $\sigma$, then $c^{f}(N, \succeq, t)=X^{n}$. We show that for each $i \in N, i \in c^{f}(N, \succeq, t)$ if and only if $i \in X^{n}$. Assume, without loss of generality, that $\sigma(i)=i$ for all $i \in \mathbb{N}$. We proceed by induction on the index of the agents. If there exists an admissible coalition $S$ such that $1 \in S$, then $X^{1}=\{S \in A C(N, \succeq, t) \mid 1 \in S\}$. In this case $1 \in X^{n}$ because $X^{n} \subseteq X^{1}$. If there does not exist an admissible coalition $S$ such that $1 \in S$, then $X^{1}=A C(N, \succeq, t)$. In this case, $1 \notin X^{n}$. Since $f$ satisfies order preservation with respect to $\sigma$, it is easy to see that $1 \in c^{f}(N, \succeq, t)$ if and only if there exists an admissible coalition $S$ such that $1 \in S$.

Assume that for all $j<i \leq n, j \in c^{f}(N, \succeq, t)$ if and only if $j \in X^{n}$. We prove that $i \in c^{f}(N, \succeq, t)$ if and only if $i \in X^{n}$. Using arguments similar to those used with agent 1 we can prove that $i \in X^{n}$ if and only if there exists an admissible coalition $S \in X^{i-1}$ such that $i \in S$. We now prove that $i \in c^{f}(N, \succeq, t)$ if and only if there exists an admissible coalition $S \in X^{i-1}$ such that $i \in S$.

Assume $i \in c^{f}(N, \succeq, t)$ and let $S=c^{f}(N, \succeq, t)$. By definition, $c^{f}(N, \succeq, t)$ is admissible. By induction hypothesis, $\{1, \ldots, i-1\} \cap c^{f}(N, \succeq, t)=\{1, \ldots, i-1\} \cap X^{n}$. Thus, $c^{f}(N, \succeq, t) \in X^{i-1}$.

Assume that there exists an admissible coalition $S \in X^{i-1}$ such that $i \in S$. By induction hypothesis, $\{1, \ldots, i-1\} \cap c^{f}(N, \succeq, t)=\{1, \ldots, i-1\} \cap X^{n}$. Since $\{1, \ldots, i\} \cap X^{n} \subset$ $S, S$ is an admissible coalition containing $\{1, \ldots, i\} \cap c^{f}(N, \succeq, t)$. Since $f$ satisfies order preservation with respect to $\sigma, i \in c^{f}(N, \succeq, t)$. 


\section{A3.2. The independence of the axioms}

Assume, by simplicity, that $\sigma(i)=i$ for all $i \in \mathbb{N}$. We define $f^{5}$ as follows. Given $S \in$ $A C(N, \succeq, t)$, define $I D_{i}^{\sigma}(S, \succeq, t)$ as the share obtained by $i$ when agents select sequentially, following the order $\sigma$, the share they prefer most corresponding to feasible and individually rational from equal division allocations (we avoid the technical definition). Given $(N, \succeq, t)$, set $c^{f^{5}}(N, \succeq, t)=c^{F^{\sigma}}(N, \succeq, t)$ and $f_{i}^{5}(N, \succeq, t)=0$ for each $i \notin c^{f^{5}}(N, \succeq, t)$ and for each $i \in c^{f^{5}}(N, \succeq, t)$

$$
f_{i}^{5}(N, \succeq, t)= \begin{cases}F_{i}^{\sigma}\left(c^{F^{\sigma}}(N, \succeq, t), \succeq_{c^{F}}(N, \succeq, t), t\right) & \text { if }\left|c^{F^{\sigma}}(N, \succeq, t)\right| \text { is odd } \\ I D_{i}^{\sigma}\left(c^{F^{\sigma}}(N, \succeq, t), \succeq_{c^{F^{\sigma}}(N, \succeq, t)}, t\right) & \text { if }\left|c^{F^{\sigma}}(N, \succeq, t)\right| \text { is even }\end{cases}
$$

It is not difficult to show that:

(1) The rule $f^{1}$ is consistent, individually rational from equal division and satisfies order preservation with respect to $\sigma$, but it is not efficient.

(2) The rule $f^{2}$ is efficient, consistent and satisfies order preservation with respect to $\sigma$, but it is not individually rational from equal division.

(3) Any extended uniform rule $F^{\sigma^{\prime}}$ with $\sigma^{\prime} \neq \sigma$ is efficient, consistent and individually rational from equal division, but it does not satisfy order preservation with respect to $\sigma$.

(4) The rule $f^{5}$ is efficient, individually rational from equal division and satisfies order preservation with respect to $\sigma$, but it is not consistent. 